\title{
Statistics of ceramic strength: Use ordinary Weibull distribution function or Weibull statistical fracture theory?
}

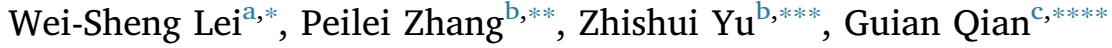 \\ a 1786 Duvall Drive, San Jose, CA 95130, USA \\ ${ }^{\mathrm{b}}$ School of Materials Engineering, Shanghai University of Engineering Science, Shanghai, 201620, China \\ ${ }^{\mathrm{c}}$ Institute of Mechanics, Chinese Academy of Sciences, Beijing, 100190, China
}

\section{A R T I C L E I N F O}

\section{Keywords:}

Strength

Ceramics

Size effect

Weibull statistics

Ordinary Weibull distribution function

Weibull statistical fracture theory

\begin{abstract}
A B S T R A C T
"Weibull statistics" for strength distribution analysis refers to either the ordinary Weibull distribution function or the Weibull statistical fracture theory. The ordinary Weibull distribution function is an empirical distribution function on an equal footing with other type of classical empirical distributions such as normal and log-normal distributions for fitting the statistical data of various random variables nonexclusive to materials strength. It has no explicit physical meaning and cannot be used for size scaling and prediction of strength. The Weibull statistical fracture theory is a weakest-link statistical fracture model for a solid with the strength distribution of an elemental volume being described by the ordinary Weibull distribution function. It has the capability of size scaling and prediction of strength for specimens with different geometries and different loading modes. The three-parameter Weibull statistical fracture theory in uniaxial flexure of prismatic beams is reformulated and validated by both numerical and real strength experiments of different ceramics.
\end{abstract}

\section{Introduction}

The strength of quasi-brittle materials [1], such as concrete [2], ceramics [3-7], and glass [8], possesses a twofold character [1-3,7-9]: First, nominally identical specimens show a large variation of fracture strength measured at same loading conditions (loading mode and loading rate). This is known as the random variation of strength. Second, there exists an inverse relationship between the nominal (average) strength and the geometrical size of specimens. This is usually called the size effect. For the random variation of strength, different ordinary statistical distribution functions ( $d f$ ), such as Weibull, normal, log-normal, exponential, and Gamma distributions, have been used to fit strength data $[5,10]$. The identification of an individual $d f$ for the "best fit" of sampled strength data relies on the goodness of fit tests for frequentist statistics such as the coefficient of determination, Akaike information criterion, the chi-square test, the Kolmogorov-Smirnov test, the Anderson-Darling test, and the Shipiro-Wilk test. Usually, the ordinary Weibull distribution [11] is most widely used [4,5,12]. The statistical approach to the size effect on strength essentially resorts to Weibull statistical fracture theory [13], as adopted by Ref. [3,7,14]. The size effect evaluation includes transference of strength data from one set of specimens to another set of specimens or real functional components (e.g. dental restorations) with either a same geometrical configuration and loading mode but different sizes or different geometries and loading modes. In literature, both the ordinary Weibull distribution and Weibull statistical fracture theory are usually called Weibull statistics.

Often, the failure of using ordinary Weibull distribution to fit a specific strength data set is attributed to the inappropriateness of Weibull statistical fracture theory [10]. Particularly, since a rationale is still lack for estimating all the three Weibull parameters, the twoparameter ordinary Weibull distribution is most frequently adopted in practice for simplicity $[4,12]$. Deng et al. [15] suggested that a threeparameter Weibull function is not necessary for analyzing the statistical variation of ceramic strength. When a set of strength data cannot be well fit by the two- or three-parameter ordinary Weibull distribution, the Weibull statistical fracture theory is often claimed invalid. Furthermore, while the two-parameter ordinary Weibull distribution is easy to calibrate, there is no consensus on a zero-valued threshold fracture strength $\left(\sigma_{L}=0\right)$ for a quasi-brittle material. From

\footnotetext{
* Corresponding author.

** Corresponding author.

*** Corresponding author.

**** Corresponding author.

E-mail addresses: leiws2008@gmail.com (W.-S. Lei), peilei@sues.edu.cn (P. Zhang), yu_zhishui@163.com (Z. Yu), qianguian@imech.ac.cn (G. Qian).
} 
experimental design aspect, strength measurement demands to test nominally identical specimens in terms of geometrical size and quality. A visual inspection of specimens prior to an experiment for the assurance of "nominal identical specimens" naturally excludes any specimen with noticeable defects such as chipping, let alone any broken specimens. That says, in a strength experiment with any number of specimens, the measured strength data are always non-zero. For example, Gorjan and Ambrožič [5] reported 5100 experimental values of the flexural strength of test pieces from a serial production of alumina ceramics, all being non-zero. According to fracture mechanics theory and experimental data, Malzbender and Steinbrech [6] justified the existence of non-zero threshold fracture strength $\left(\sigma_{L} \neq 0\right)$ of $1 \mathrm{~mm}$ thick porous ceramic components in biaxial flexure. In the case that no processing related cracks are introduced, the size of defects such as pores are limited by specimen thickness. Then for a thin ceramic specimen in biaxial flexure, there exists a non-zero threshold strength. Smart et al. [16] concluded the necessity to test a very large number of specimens to have any certainty whether the strength data belong to a two- or threeparameter ordinary Weibull distribution. Usually, using the two-parameter ordinary Weibull distribution to fit strength data yields much different values of Weibull modulus and scale parameters for specimens made of same material but with different geometries and loading modes [17]. This is against the common assumption that the Weibull parameters are materials property independent of specimen geometry and loading mode. In consequence, it brings the challenges on how to transfer strength data from one specimen geometry to another with same or different loading mode, and more importantly, on how to predict fracture occurrence of a real structural component with complex geometry such as a dental restoration (e.g., crown, fixed dental prosthesis frame) based upon laboratory collected strength data from specimens with a simple geometry under a simple loading condition.

The above discussion calls for a clarification on the difference between the ordinary Weibull distribution [11] and Weibull statistical fracture theory [13] for strength analysis.

In the proceeding sections, first, the distinction between the ordinary Weibull distribution [11] and Weibull statistical fracture theory [13] will be elaborated. Next, a detailed analysis of Weibull statistical fracture theory is provided in uniaxial flexure. The cumulative failure probability in uniaxial flexure is reformulated, which raises the cautions to distinguish between the three-parameter ordinary Weibull distribution function and the three-parameter Weibull statistical strength theory. A method of strength data fitting based on Weibull statistical fracture theory is introduced and compared with that based on the ordinary Weibull distribution.

\section{The difference between Weibull statistical theory of strength and ordinary Weibull distribution function}

Although it is often ignored, the distinction between the ordinary Weibull distribution function and Weibull statistical theory of strength has been clearly highlighted by the titles of Weibull's two eminent papers $[11,13]$ as below:

(1). A statistical theory of the strength of materials [13];

(2). A statistical distribution function of wide applicability [11].

At first, Weibull [13] proposed the following cumulative failure probability formulation for a bulk material with a uniform spatial distribution of flaws based on the weakest link postulate and the maximum tensile principal stress $\left(\sigma_{1}\right)$ criterion for fracture $\sigma_{1}=\sigma \geq S$ :

$$
\begin{aligned}
P\left(\sigma_{N}\right)= & 1-\exp [-B]=1 \\
& -\exp \left[-\int_{V}\left(\frac{\sigma_{1}-\sigma_{L}}{\sigma_{u}}\right)^{m} \frac{\delta V}{V_{0}}\right] \quad\left(\sigma_{L} \leq \sigma_{1}<\infty\right)
\end{aligned}
$$

$B=\ln \left[\frac{1}{1-P\left(\sigma_{N}\right)}\right]=\int_{V}\left(\frac{\sigma_{1}-\sigma_{L}}{\sigma_{0}}\right)^{m} \frac{d V}{V_{0}}$

where $P\left(\sigma_{N}\right)$ is the cumulative probability of failure under the external loading measured by a nominal stress $\sigma_{N}, \sigma_{u}$ is a scale parameter independent of specimen size, $\sigma_{L}$ is a threshold strength below which fracture does not occur, $m$ is the shape parameter, $\sigma_{1}$ is the maximum tensile principal stress at any point inside a specimen of volume $V$ due to the external loading, $V_{O}$ is a reference volume, $d V$ is a differential volume element. $B$ is the risk of rupture defined by Weibull [13].

For a detailed interpretation of Equation (1), a revisit to Weibull statistical fracture theory [13] was made in Lei [18,19], along with a reformulated weakest-link formulation for fracture. By assuming a uniform spatial distribution of flaws in a material, the following cumulative probability of failure was formulated for the weakest link postulate $[18,19]$ :

$P(V)=1-\exp \left\{\int_{V} \ln \left[1-p\left(\sigma, V_{0}\right)\right] \frac{d V}{V_{0}}\right\}$

where $p\left(\sigma, V_{0}\right)$ is the fracture probability of an elemental volume $\mathrm{V}_{0}$ with an embedded flaw of size $a$ under stress $\sigma$.

When the flaw distribution in terms of strength (S) of an elemental volume $\mathrm{V}_{0}$ is described by the probability density function (PDF) below:

$g(S)=\frac{m}{\sigma_{u}}\left(\frac{S-\sigma_{L}}{\sigma_{u}}\right)^{m-1} \exp \left[-\left(\frac{S-\sigma_{L}}{\sigma_{u}}\right)^{m}\right]$

the maximum tensile principal stress criterion $\left(\sigma_{1}=\sigma \geq S\right)$ for unstable fracture leads to,

$p\left(\sigma, V_{0}\right)=\int_{\sigma_{L}}^{\sigma} g(S) d S=1-\exp \left[-\left(\frac{\sigma-\sigma_{L}}{\sigma_{u}}\right)^{m}\right]\left(\sigma_{L} \leq \sigma<\infty\right)$

or in equivalence,

$\ln \left[\frac{1}{1-p\left(\sigma, V_{0}\right)}\right]=\left(\frac{\sigma-\sigma_{L}}{\sigma_{u}}\right)^{m}\left(\sigma_{L} \leq \sigma<\infty\right)$

Note here all the three material parameters $\sigma_{L}, \sigma_{u}$, and $m$ are constant. Substitution of Equation (5) or (6) in Equation (3) leads to Equation (1) for the Weibull statistical fracture theory. It is worth mentioning that the adoption of Equation (4) as the flaw distribution only warrants the mathematical self-consistency of Weibull statistical fracture theory [13] in Equation (1) built on the weakest-link formulation in Equation (3). By no means it claims that Equation (4) dictates the flaw distribution in any material. When a different flaw distribution function $g(S)$ is adopted, according to Equation (5), the fracture probability of an elemental volume $\mathrm{V}_{0}$ with an embedded flaw of size $a$ under stress $\sigma, p\left(\sigma, V_{0}\right)$, as an integral of $g(S)$ in the loading regime $\left[\sigma_{L}, \sigma\right]$, will have a different expression, leading to a non-Weibull type, often more complicated mathematical expression of the cumulative failure probability $P$ according to Equation (3). This will be further elaborated in Section 5 Discussions. A comparison of Equations (1) and (2) and Equation (6) highlights B as "the risk of rupture" defined by Weibull [13]:

$\mathrm{B}_{0}=\ln \left[\frac{1}{1-p\left(\sigma, V_{0}\right)}\right], \ldots B=\ln \left[\frac{1}{1-P(\sigma, V)}\right]$,

Equation (1) can be rewritten as

$P\left(\sigma_{N}\right)=1-\exp \left[-\left(\frac{\sigma_{N}-\sigma_{L}}{\sigma_{0}}\right)^{m}\right]$ 


$$
\begin{aligned}
\sigma_{0}= & \frac{\sigma_{u}}{\left[\int_{V}\left(\frac{\sigma_{1}-\sigma_{L}}{\sigma_{N}-\sigma_{L}}\right)^{m} \frac{d V}{V_{0}}\right]^{1 / m}}= \\
& \left\{\begin{array}{cc}
\sigma_{u}\left(V_{0} / V\right)^{1 / m} & \text { (uniaxial tension) } \\
\delta \cdot \sigma_{u}\left(V_{0} / V\right)^{1 / m} & \left(\sigma_{L}=0\right) \\
h\left(\sigma_{N}: m, \sigma_{L}, \sigma_{u}, V_{0} / V\right) \cdot \sigma_{u} & \left(\sigma_{L} \neq 0: \text { non }- \text { uniform stress }\right)
\end{array}\right.
\end{aligned}
$$

where $\sigma_{0}$ is a specimen size dependent scale parameter, $\delta=\left[\int_{V}\left(\sigma_{1} / \sigma_{N}\right)^{m} d V / V\right]^{1 / m}$ as a coefficient is independent of nominal stress $\sigma_{N}$ but varies with specimen geometry and loading mode, $h\left(\sigma_{N}: m, \sigma_{L}, \sigma_{u}, V_{0} / V\right)=\left[\int_{V}\left(\frac{\sigma_{1}-\sigma_{L}}{\sigma_{N}-\sigma_{L}}\right)^{m} \frac{d V}{V_{0}}\right]^{-1 / m}$ is a function of nominal stress $\sigma_{N}$, specimen geometry and size $(V)$, and loading mode.

For Weibull statistical fracture theory, there are two key takeaways:

(1). Equation (1) is the basic formulation of Weibull statistical fracture theory, also known as Weibull statistical theory for strength;

(2). Unlike the scale parameter $\sigma_{u}$ with a constant value, the parameter $\sigma_{0}$ varies with specimen geometry and loading mode. For the uniaxial smooth tension (Equation (9a)) the parameter $\sigma_{0}$ depends on specimen volume $(\mathrm{V})$; for $\sigma_{L}=0$ in any loading mode (Equation (9b)), the parameter $\sigma_{0}$ varies with specimen volume and loading mode; In all other cases (Equation (9c), the scale parameter $\sigma_{0}$ varies with specimen volume $(\mathrm{V})$, loading mode, and the nominal stress $\sigma_{N}$.

20 years later, based on Equation (8), Weibull [11] proposed the following more general expression as an empirical distribution function for a broad range of applications, which include but are not limited to the field of applied mechanics, such as the yield strength and the fatigue life of steels, the size distribution of fly fish, the length of Cyrtoideae, the statures for adult males born in the British Isles, and the breadth of common beans.

$F(x)=P(X \leq x)=1-\exp \left[-\left(\frac{x-x_{L}}{x_{0}}\right)^{m}\right]$

where $\mathrm{F}(x)$ is the $d f$, or more accurately, the cumulative distribution function (CDF) of a random variable $X$, which defines the probability of the random variable $X$ having a value not exceeding $x, P(X \leq x), x_{0}$ is a scale parameter of constant value, $x_{L}$ is a threshold value so that $x \geq x_{L}$. form

Weibull [15] justified Equation (10) by writing any $d f F(x)$ in the

$F(x)=1-\exp [-\phi(x)]$

where the function $\phi(x)$ is to be specified. The normative axiom of probability demands that the function $F(x)$ obeys $0 \leq F(x) \leq 1$. Therefore, Weibull pointed out that "The only necessary general condition this function has to satisfy is to be a positive, non-decreasing function, vanishing at a value $\left(x_{L}\right)$, which is not of necessity equal to zero". Weibull came up with "the simplest function satisfying this condition" as

$\phi(x)=\left(\frac{x-x_{L}}{x_{0}}\right)^{m}$

Substitution of Equation (12) in Equation (11) gives Equation (10). Equation (10) is known as the ordinary Weibull distribution function. Weibull [11] noted that "The objection has been stated that this distribution function has no theoretical basis. But in so far as the author understands, there are-with very few exceptions-the same objections against all other df, applied to real populations from natural or biological fields, at least in so far as the theoretical basis has anything to do with the population in question".

Therefore, the ordinary Weibull distribution in Equation (10) should be considered as an empirical one on an equal footing with other type of classical ordinary distributions such as normal, log-normal, power law, and Gamma distributions. All these empirical distribution functions do not necessarily have any theoretical basis. Today, the ordinary Weibull distribution Equation (10) has been used in many other fields such as the liquid drop size distribution in the liquid-liquid extraction process for chemical separation applications [20] and wind speed and wind energy distributions in renewable energy industry [21].

This short visit to Weibull's two papers [11,13] leads to the following observations:

(1). Equation (1) as the basic formulation of Weibull statistical fracture theory is based on the weakest link postulate, while Equation (10) as the ordinary Weibull distribution function is only an empirical distribution function that has nothing to do with the weakest link postulate. Unlike other random variables such as wind speed which can be analyzed by ordinary Weibull distribution, coincidentally, the strength of quasi-brittle materials can be analyzed by either the ordinary Weibull distribution function as an empirical approach or Weibull statistical fracture theory in Equation (1).

(2). The ordinary Weibull distribution function [11] in Equation (10) is an empirical extension of the strength distribution function in Equation (8) to the distribution of other random variables under the assumption of a constant-valued scale parameter $\left(\sigma_{0}\right.$ or $\left.x_{0}\right)$. In Weibull statistical fracture theory [13], as shown in Equation (9c), while the first scale parameter $\sigma_{u}$ is a material property of constant value, the second scale parameter $\sigma_{0}$ is not. The conventional practice of using Equation (10) to fit strength data naturally assumes a constant value to the second scale parameter $\sigma_{0}$ in Equation (8). Therefore, the calibrated Weibull parameters is only valid for a given specimen geometry and size with a specific loading mode. That says, while it is used for strength data fitting, the ordinary Weibull distribution function Equation (10) is incapable of characterizing the size dependence of strength. In contrast, Equation (1) for the Weibull statistical fracture theory can address both aspects.

\section{Weibull statistical fracture theory in uniaxial tension and uniaxial flexure}

\subsection{Uniaxial tension}

Under uniaxial tension, Equation (1) is simplified as

$$
\begin{aligned}
P(\sigma, V)= & 1-\exp \left[-\left(\frac{\sigma-\sigma_{L}}{\sigma_{u}}\right)^{m} \frac{V}{V_{0}}\right]=1 \\
& -\exp \left[-\left(\frac{\sigma-\sigma_{L}}{\sigma_{0}}\right)^{m}\right] \quad\left(\sigma \geq \sigma_{L}\right)
\end{aligned}
$$

with $\sigma_{0}=\sigma_{u}\left(V_{0} / V\right)^{1 / m}$, which is specimen size (V) dependent. Due to the preceding correlation between $\sigma_{\mathrm{u}}$ and $\sigma_{0}$, the value of $\sigma_{0}$ via data fitting according to the ordinary Weibull distribution function Equation (10) is not an intrinsic material property but varies with the intrinsic parameters ( $\sigma \mathrm{u}, \mathrm{m})$ and the volume $(\mathrm{V})$ of a specimen.

\subsection{Uniaxial flexure}

Consider a prismatic beam in four-point flexure (Fig. 1). The uniaxial tensile stress $\sigma(x, y)$ at any point $(x, y)$ in the prismatic beam follows

$\frac{\sigma(x, y)}{\sigma_{p}}=\left\{\begin{array}{cc}\frac{2 k x y}{h L} & \left(x \in\left[0, \frac{L}{k}\right], x \in\left[\left(1-\frac{1}{k}\right) L, L\right], y \in\left[0, \frac{h}{2}\right]\right) \\ \frac{2 y}{h} & \left(x \in\left[\frac{L}{k},\left(1-\frac{1}{k}\right) L\right], y \in\left[0, \frac{h}{2}\right]\right)\end{array}\right.$

where $\sigma_{\mathrm{p}}$ is the peak stress value at the outer fiber, which is taken as the nominal flexural strength, $\sigma_{\mathrm{N}}=\sigma_{\mathrm{p}}, b$ is the beam width, $h$ is the beam 


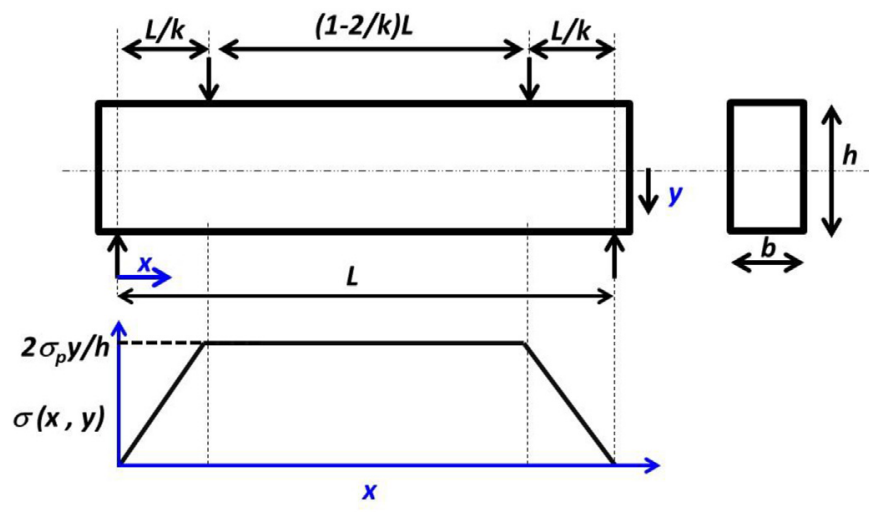

Fig. 1. Schematic setup of a prismatic beam in four-point flexure and tensile stress distribution in the beam, with $\mathrm{k}=\infty$ for pure bending; $\mathrm{k}=4$ for fourpoint bending; $\mathrm{k}=2$ for three-point bending.

height, $L$ is the (outer) support span, $(1-2 / k) L$ is the (inner) loading span. $k$ is an integer. Specifically, $k=\infty$ corresponds to pure bending (PB); $k=4$ is for four-point bending (4PB); $k=2$ refers to three-point bending (3PB).

Weil and Daniel [22] reported the analytical solutions to the risk of rupture (B) defined in Equation (2) for fracture induced by volumetric and surficial flaws, respectively. Refer to Fig. 1, for fracture controlled by volumetric flaws,

$$
\begin{aligned}
B= & \frac{V}{2(m+1) V_{0}}\left(1-\frac{\sigma_{L}}{\sigma_{p}}\right)\left(\frac{\sigma_{p}-\sigma_{L}}{\sigma_{u}}\right)^{m} \\
& {\left[\left(1-\frac{2}{k}\right)+\frac{2}{k} \sum_{r=0}^{[m]} \frac{1}{m+1+r}\left(1-\frac{\sigma_{p}}{\sigma_{L}}\right)^{-r}\right] } \\
& +\frac{I(\alpha) V}{k(m+1) V_{0}} \frac{\left(-\sigma_{L}\right)^{[m]+1}}{\sigma_{p} \sigma_{u}^{m}}
\end{aligned}
$$

with

$I(\alpha)=\int_{\sigma_{L} / \sigma_{p}}^{1}\left(\sigma_{p} Z-\sigma_{L}\right)^{\alpha} \frac{d Z}{Z}$

where $m=[m]+\alpha,[m]$ is the maximum integer less than or equal to $m$, so $0 \leq \alpha<1, V=b h L$.

When $m$ is an integer, $m=[m], \alpha=0, I(\alpha=0)=\ln \left(\sigma_{b} / \sigma_{L}\right)$, Equation (15) reduces to

$$
\begin{aligned}
B= & \frac{V}{2(m+1) V_{0}}\left(1-\frac{\sigma_{L}}{\sigma_{p}}\right)\left(\frac{\sigma_{p}-\sigma_{L}}{\sigma_{u}}\right)^{m} \\
& {\left[\left(1-\frac{2}{k}\right)+\frac{2}{k} \sum_{r=0}^{m} \frac{1}{m+1+r}\left(1-\frac{\sigma_{p}}{\sigma_{L}}\right)^{-r}\right] } \\
& +\frac{V}{k(m+1) V_{0}} \frac{\left(-\sigma_{L}\right)^{m+1}}{\sigma_{p} \sigma_{u}^{m}} \ln \left(\frac{\sigma_{p}}{\sigma_{L}}\right)
\end{aligned}
$$

When $\sigma_{\mathrm{L}}=0$, Equation (15) reduces to

$B=K \frac{V}{V_{0}}\left(\frac{\sigma_{p}}{\sigma_{u}}\right)^{m}=\frac{k(m+1)-2 m}{2 k(m+1)^{2}} \frac{V}{V_{0}}\left(\frac{\sigma_{p}}{\sigma_{u}}\right)^{m}$

with

$K=\frac{k(m+1)-2 m}{2 k(m+1)^{2}}$

Two key points are taken from Weibull statistical fracture theory for the uniaxial flexure:

(1). The three-parameter ordinary Weibull distribution function Equation (10) does not have a physical connection with the distribution of flexural strength represented by the combination of Equations (1) and (15).

(2). The two-parameter ordinary Weibull distribution function is in line with Weibull statistical fracture theory for $\sigma_{L}=0$ in uniaxial tension or flexure, except that $\sigma_{O}$ is specimen size (V) and loading mode $(k)$ dependent.

\subsection{Reformulated Weibull statistical fracture theory for uniaxial flexure}

The solutions by Weil and Daniel [22] are suitable for $m \geq 1$. Technically, when $\mathrm{m}$ is not an integer, the solutions for $\mathrm{m}$ can be estimated via the interpolation method from the results for the integers $[\mathrm{m}]$ and $[\mathrm{m}+1]$. In addition, the solutions for $\mathrm{m}$ as an integer contain the logarithmic quantity $\ln \left(\frac{\sigma_{p}}{\sigma_{L}}\right)$, which is inconvenient to use. Therefore, the solutions to the risk of rupture (B) are redeveloped in this section for $\mathrm{m}$ as a positive integer to get rid of the logarithmic quantity $\ln \left(\frac{\sigma_{p}}{\sigma_{L}}\right)$.

\subsubsection{Reformulation process}

Also refer to Fig. 1 and assume $\mathrm{m}$ as a positive integer. According to Equation (1), there is

$P\left(\sigma_{N}\right)=1-\exp [-B]=1-\exp \left[-\left(B_{1}+B_{2}\right)\right] \quad\left(\sigma_{L} \leq \sigma_{1}<\infty\right)$

with

$B=\int_{V}\left(\frac{\sigma-\sigma_{L}}{\sigma_{u}}\right)^{m} \frac{d V}{V_{0}}=B_{1}+B_{2}$

$B_{1}=\frac{2 b}{V_{0}} \int_{y_{\min }}^{h / 2} d y \int_{x_{\min }}^{x_{\max }}\left(\frac{\frac{2 k \sigma_{p}}{h L} y x-\sigma_{L}}{\sigma_{u}}\right)^{m} d x$

$B_{2}=\frac{b}{V_{0}} \int_{L / k}^{1-L / k} d x \int_{y_{\min }}^{h / 2}\left(\frac{\frac{2 \sigma_{p}}{h} y-\sigma_{L}}{\sigma_{u}}\right)^{m} d y$

Denote that $\mathrm{X}=\frac{x}{L / k}, Y=\frac{y}{h / 2}, T=\frac{\sigma_{L}}{\sigma_{p}}$. Now Equation (22) reduces to $B_{1}=\frac{V}{k V_{0}} \cdot\left(\frac{\sigma_{p}}{\sigma_{u}}\right)^{m} \int_{T}^{1} \frac{d Y}{Y} \int_{T}^{1}[X Y-T]^{m} d(X Y-T)$

or

$B_{1}=\frac{1}{k(m+1)} \cdot \frac{V}{V_{0}} \cdot\left(\frac{\sigma_{p}}{\sigma_{u}}\right)^{m} \int_{T}^{1} \frac{d Y}{Y}\left[(Y-T)^{m+1}-(T Y-T)^{m+1}\right]$

With $\mathrm{m}$ being a positive integer, by factoring the polynomial $\left(a^{m}-b^{m}\right)$ and expanding $(x-y)^{m}$, Equation (25) is rewritten as

$B_{1}=\frac{1}{k(m+1)} \cdot \frac{V}{V_{0}} \cdot\left(\frac{\sigma_{p}}{\sigma_{u}}\right)^{m}(1-T)^{m+2} \sum_{i=0}^{m} T^{i} \sum_{j=0}^{i}\left(\begin{array}{l}i \\ j\end{array}\right) \frac{(-1)^{i-j}}{m+j-i+1}$

or

$$
B_{1}=\frac{1}{k(m+1)} \cdot \frac{V}{V_{0}} \cdot\left(1-\frac{\sigma_{L}}{\sigma_{p}}\right)^{2}\left(\frac{\sigma_{p}-\sigma_{L}}{\sigma_{u}}\right)^{m} \sum_{i=0}^{m} T^{i} \sum_{j=0}^{i}\left(\begin{array}{l}
i \\
j
\end{array}\right) \frac{(-1)^{i-j}}{m+j-i+1}
$$

Here $\left(\begin{array}{l}i \\ j\end{array}\right)=\frac{i !}{j !(i-j) !}$ is a binomial coefficient.

The expressions of $\sum_{i=0}^{m} T^{i} \sum_{j=0}^{i}\left(\begin{array}{l}i \\ j\end{array}\right) \frac{(-1)^{i-j}}{m+j-i+1}$ are listed in Appendix A for $m=1,2,3, \ldots, 10$, respectively. It can be proven that, 


$$
\begin{aligned}
\sum_{i=0}^{m} T^{i} \sum_{j=0}^{i}\left(\begin{array}{l}
i \\
j
\end{array}\right) \frac{(-1)^{i-j}}{m+j-i+1} & =\frac{1}{m+1}+O\left(\frac{1}{m(m+1)}\right) \cdot \frac{\sigma_{L}}{\sigma_{p}}=\frac{1}{m+1} \\
& {\left[1+O\left(\frac{1}{m(m+1)^{2}} \cdot \frac{\sigma_{L}}{\sigma_{p}}\right)\right] }
\end{aligned}
$$

Here $O(x)$ denotes a quantity smaller in absolute value than $x$. For $m \geq 5$, Equation (28) takes the following approximation with an error $\leq 5 \%$ :

$\sum_{i=0}^{m} T^{i} \sum_{j=0}^{i}\left(\begin{array}{l}i \\ j\end{array}\right) \frac{(-1)^{i-j}}{m+j-i+1} \approx \frac{1}{m+1} \ldots(m \geq 5)$

Due to Equation (29), Equation (27) may further reduce to

$B_{1}=\frac{1}{k(m+1)^{2}} \cdot \frac{V}{V_{0}} \cdot\left(1-\frac{\sigma_{L}}{\sigma_{p}}\right)^{2}\left(\frac{\sigma_{p}-\sigma_{L}}{\sigma_{u}}\right)^{m}\left[1+O\left(\frac{1}{m(m+1)^{2}} \cdot \frac{\sigma_{L}}{\sigma_{p}}\right)\right]$

Similarly, the quantity $\mathrm{B}_{2}$ defined by Equation (23) is

$B_{2}=\frac{V}{2 k V_{0}}\left(\frac{\sigma_{p}}{\sigma_{0}}\right)^{m} \int_{1}^{(k-1)} d X \int_{T}^{1}[Y-T]^{m} d(Y-T)$

or

$B_{2}=\frac{(k-2)}{2(m+1) k} \cdot \frac{V}{V_{0}} \cdot\left(1-\frac{\sigma_{L}}{\sigma_{p}}\right)\left(\frac{\sigma_{p}-\sigma_{L}}{\sigma_{u}}\right)^{m}$

Inserting Equations (30) and (32) into Equation (21) yields

$$
\begin{aligned}
B= & \frac{1}{(m+1)} \cdot \frac{V}{V_{0}} \\
& \cdot\left(1-\frac{\sigma_{L}}{\sigma_{p}}\right)\left(\frac{\sigma_{p}-\sigma_{L}}{\sigma_{u}}\right)^{m}\left\{\frac{1}{k(m+1)} \cdot\left(1-\frac{\sigma_{L}}{\sigma_{p}}\right)\right. \\
& {\left.\left[1+O\left(\frac{1}{m(m+1)^{2}} \cdot \frac{\sigma_{L}}{\sigma_{p}}\right)\right]+\frac{(k-2)}{2 k}\right\} }
\end{aligned}
$$

Particularly, there are ( $m$ being an integer):

(1). $k=\infty$ : pure bending (PB),

$B_{1}=0$

$B=B_{2}=\frac{1}{2(m+1)} \cdot \frac{V}{V_{0}} \cdot\left(1-\frac{\sigma_{L}}{\sigma_{p}}\right)\left(\frac{\sigma_{p}-\sigma_{L}}{\sigma_{u}}\right)^{m}$

(2). $k=2$ : three-point bending (3PB),

$B_{2}=0$

$B=B_{1}=\frac{1}{2(m+1)^{2}} \cdot \frac{V}{V_{0}}$

$$
\cdot\left(1-\frac{\sigma_{L}}{\sigma_{p}}\right)^{2}\left(\frac{\sigma_{p}-\sigma_{L}}{\sigma_{u}}\right)^{m}\left[1+O\left(\frac{1}{m(m+1)^{2}} \cdot \frac{\sigma_{L}}{\sigma_{p}}\right)\right]
$$

(3). $k=4$ : four-point bending (4PB),

$B_{1}=\frac{1}{4(m+1)^{2}} \cdot \frac{V}{V_{0}} \cdot\left(1-\frac{\sigma_{L}}{\sigma_{p}}\right)^{2}\left(\frac{\sigma_{p}-\sigma_{L}}{\sigma_{u}}\right)^{m}\left[1+O\left(\frac{1}{m(m+1)^{2}} \cdot \frac{\sigma_{L}}{\sigma_{p}}\right)\right]$

$B_{2}=\frac{1}{4(m+1)} \cdot \frac{V}{V_{0}} \cdot\left(1-\frac{\sigma_{L}}{\sigma_{p}}\right)\left(\frac{\sigma_{p}-\sigma_{L}}{\sigma_{u}}\right)^{m}$

$$
\begin{aligned}
B= & \frac{1}{4(m+1)} \cdot \frac{V}{V_{0}} \cdot\left(1-\frac{\sigma_{L}}{\sigma_{p}}\right)\left(\frac{\sigma_{p}-\sigma_{L}}{\sigma_{u}}\right)^{m}+\frac{1}{4(m+1)^{2}} \cdot \frac{V}{V_{0}} \\
& \cdot\left(1-\frac{\sigma_{L}}{\sigma_{p}}\right)^{2}\left(\frac{\sigma_{p}-\sigma_{L}}{\sigma_{u}}\right)^{m}\left[1+O\left(\frac{1}{m(m+1)^{2}} \cdot \frac{\sigma_{L}}{\sigma_{p}}\right)\right]
\end{aligned}
$$

Especially, when $\sigma_{\mathrm{L}}=0$, Equation (33) reduces to Equation (19) given by Weil and Daniel [26].

\subsubsection{Further approximations}

For pure bending, substitution of Equations (34) and (35) in Equation (20) yields:

$$
\begin{aligned}
P= & 1-\exp \left[-\frac{1}{2(m+1)} \cdot \frac{V}{V_{0}} \cdot\left(1-\frac{\sigma_{L}}{\sigma_{p}}\right)\left(\frac{\sigma_{p}-\sigma_{L}}{\sigma_{u}}\right)^{m}\right]=1 \\
& -\exp \left[-\frac{1}{(m+1)} \cdot \frac{V_{E, P B}}{V_{0}}\left(\frac{\sigma_{p}-\sigma_{L}}{\sigma_{u}}\right)^{m}\right]
\end{aligned}
$$

Here $V_{E, P B}=\frac{V}{2}\left(1-\frac{\sigma_{L}}{\sigma_{p}}\right)$ is the volume of fracture process zone in pure bending (PB) where $\sigma(x, y) \geq \sigma_{L}$ is satisfied.

Three-point bending(3PB). Based on Equation (37), the following approximate expression is introduced

$$
\begin{aligned}
P \approx & 1-\exp \left[-\frac{1}{2(m+1)^{2}} \cdot \frac{V}{V_{0}} \cdot\left(1-\frac{\sigma_{L}}{\sigma_{p}}\right)^{2}\left(\frac{\sigma_{p}-\sigma_{L}}{\sigma_{u}}\right)^{m}\right]=1 \\
& -\exp \left[-\frac{1}{(m+1)^{2}} \cdot \frac{V_{E, 3 P B}}{V_{0}}\left(\frac{\sigma_{p}-\sigma_{L}}{\sigma_{u}}\right)^{m}\right]
\end{aligned}
$$

Here $V_{E, 3 P B}=\frac{V}{2}\left(1-\frac{\sigma_{L}}{\sigma_{P}}\right)^{2}$ is the volume of fracture process zone in 3PB, in which $\sigma(x, y) \geq \sigma_{L}$ is satisfied. In case the quantity $\left(1-\frac{\sigma_{L}}{\sigma_{p}}\right)^{2} \approx 1$ is taken, the following expression will be reached, which is similar to Equation (10) for the ordinary three-parameter Weibull distribution function except that $\sigma_{0}$ varies with specimen size:

$$
\begin{aligned}
P= & 1-\exp \left[-\frac{1}{2(m+1)^{2}} \cdot \frac{V}{V_{0}} \cdot\left(\frac{\sigma_{p}-\sigma_{L}}{\sigma_{u}}\right)^{m}\right]=1 \\
& -\exp \left[-\left(\frac{\sigma_{p}-\sigma_{L}}{\sigma_{0}}\right)^{m}\right] \ldots\left(\sigma_{0}=\sigma_{u} \cdot \sqrt[m]{2(m+1)^{2}\left(V_{0} / V\right)}\right)
\end{aligned}
$$

Fig. 2 compares Equations (42) and (43) against Equation (37)for different combinations of $\mathrm{m}=5,10, \mathrm{~V} / \mathrm{V}_{0}=1,1000,10000$, and $\sigma_{\mathrm{L}} /$ $\sigma_{\mathrm{u}}=0.25,0.5$ at $\sigma_{\mathrm{u}}=600 \mathrm{MPa}$. Clearly, Equation (42) is a close approximation to Equation (37), while Equation (43) gives unacceptable approximation. This clearly reveals the inappropriateness of applying the ordinary three-parameter Weibull distribution function Equation (10) for statistical characterization of uniaxial flexural strength.

Four-point bending(4PB). Due to Equation (40), the following two approximate expressions are introduced:

$P \approx 1$

$$
\begin{aligned}
& -\exp \left[-\frac{1}{4(m+1)^{2}} \cdot \frac{V}{V_{0}} \cdot\left(1-\frac{\sigma_{L}}{\sigma_{p}}\right)^{2}\left(\frac{\sigma_{p}-\sigma_{L}}{\sigma_{u}}\right)^{m}-\frac{1}{4(m+1)} \cdot \frac{V}{V_{0}}\right. \\
& \left.\left(1-\frac{\sigma_{L}}{\sigma_{p}}\right)\left(\frac{\sigma_{p}-\sigma_{L}}{\sigma_{u}}\right)^{m}\right]
\end{aligned}
$$

$$
\begin{aligned}
P \approx & 1-\exp \left[-\frac{1}{4(m+1)} \cdot \frac{V}{V_{0}} \cdot\left(1-\frac{\sigma_{L}}{\sigma_{p}}\right)\left(\frac{\sigma_{p}-\sigma_{L}}{\sigma_{u}}\right)^{m}\right]=1 \\
& -\exp \left[-\frac{1}{2(m+1)} \cdot \frac{V_{E, P B}}{V_{0}}\left(\frac{\sigma_{p}-\sigma_{L}}{\sigma_{u}}\right)^{m}\right]
\end{aligned}
$$

Equation (45) approximates four-point bending (4PB) into pure bending (PB) by omitting the higher order term with $\left(1-\frac{\sigma_{L}}{\sigma_{P}}\right)^{2}$ in 

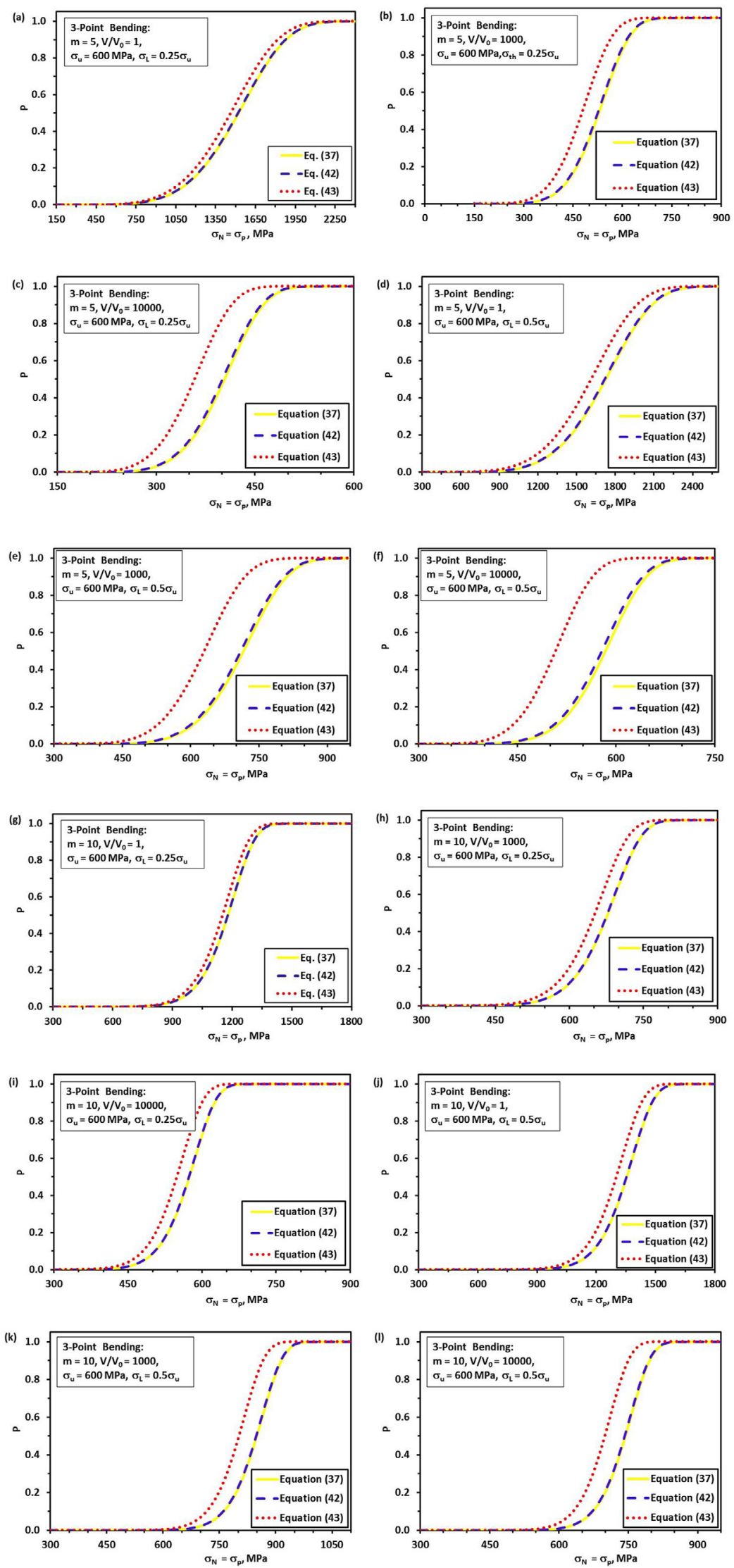

Fig. 2. Illustration of Equation (42) as a close approximation to Equation (37) in three-point bending with different combinations of $\mathrm{m}$, V/ $\mathrm{V}_{0}$ and $\sigma_{\mathrm{L}} / \sigma_{\mathrm{u}}$. 

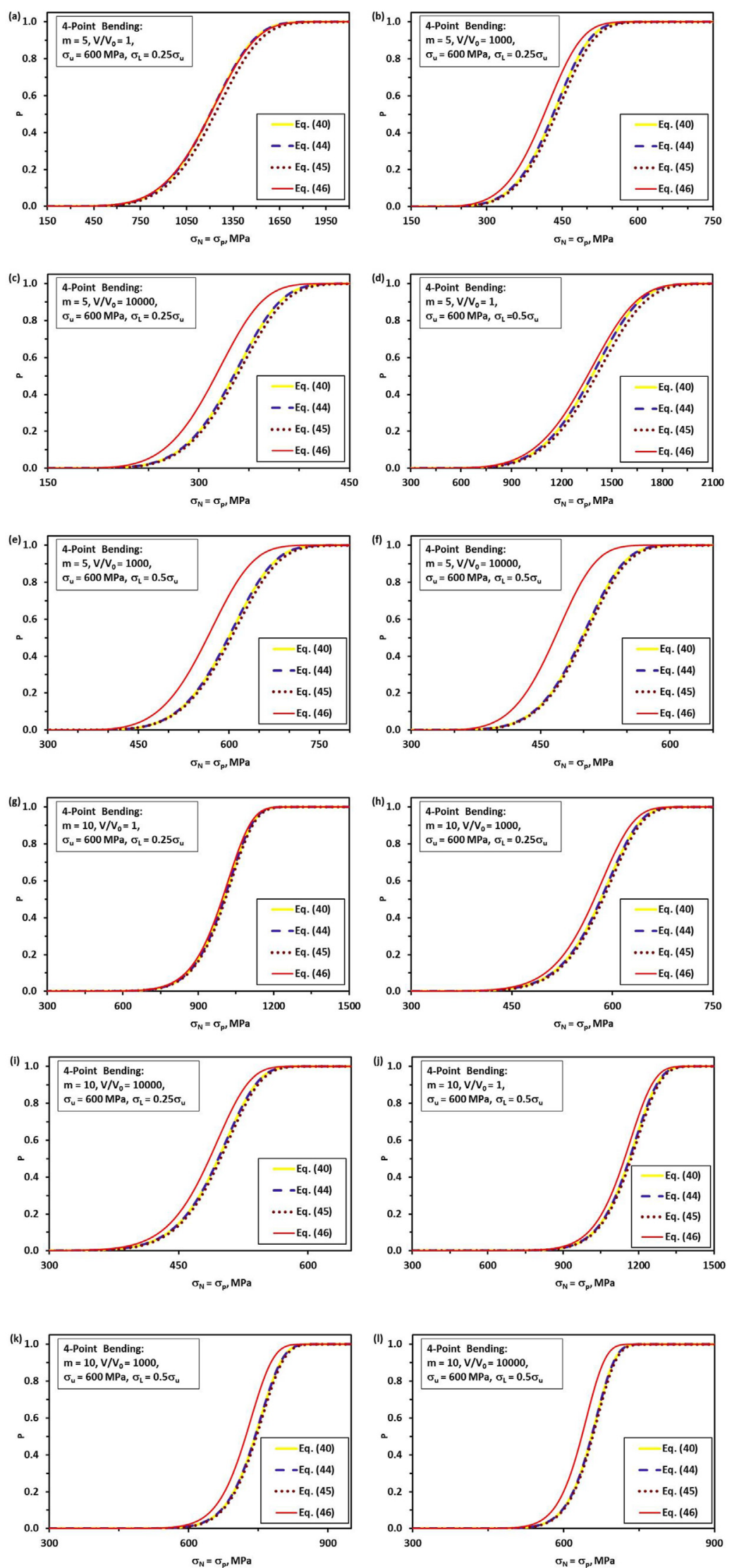

Fig. 3. Illustration of Equations (44) and (45) as close approximations to Equation (40) in combination with Equation (20) in four-point bending with different combinations of $\mathrm{m}, \mathrm{V} / \mathrm{V}_{0}$, and $\sigma_{\mathrm{L}} / \sigma_{\mathrm{u}}$ 
Table 1

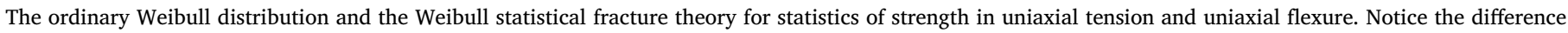
between $\sigma_{0}$ and $\sigma_{u}$.

\begin{tabular}{|c|c|c|c|c|}
\hline \multirow[t]{2}{*}{ Threshold strength $\sigma_{\mathrm{L}}$} & \multirow[t]{2}{*}{ Type of experiment $\left(\sigma_{N}=\sigma_{p}\right)$} & \multirow{2}{*}{$\begin{array}{l}\text { Volume of fracture process zone } \\
V_{E}\end{array}$} & \multicolumn{2}{|l|}{ Risk of rupture B = Ln [1/(1- P)] } \\
\hline & & & Weibull statistical fracture theory & Ordinary Weibull distribution \\
\hline \multirow[t]{4}{*}{$\sigma_{L} \neq 0$} & Uniaxial tension & $V$ & $\frac{V}{V_{0}}\left(\frac{\sigma_{p}-\sigma_{L}}{\sigma_{u}}\right)^{m}$ & $\left(\frac{\sigma_{p}-\sigma_{L}}{\sigma_{0}}\right)^{m}$ \\
\hline & Pure bending & $\frac{V}{2}\left(1-\frac{\sigma_{L}}{\sigma_{p}}\right)$ & $\frac{1}{2(m+1)} \cdot \frac{V}{V_{0}} \cdot\left(1-\frac{\sigma_{L}}{\sigma_{p}}\right)\left(\frac{\sigma_{p}-\sigma_{L}}{\sigma_{u}}\right)^{m}$ & \\
\hline & 3-point bending & $\frac{V}{2}\left(1-\frac{\sigma_{L}}{\sigma_{p}}\right)^{2}$ & $\frac{1}{2(m+1)^{2}} \cdot \frac{V}{V_{0}} \cdot\left(1-\frac{\sigma_{L}}{\sigma_{p}}\right)^{2}\left(\frac{\sigma_{p}-\sigma_{L}}{\sigma_{U}}\right)^{m}$ & \\
\hline & 4-point bending* & $\frac{V}{4}\left(1-\frac{\sigma_{L}}{\sigma_{p}}\right)+\frac{V}{4}\left(1-\frac{\sigma_{L}}{\sigma_{p}}\right)^{2} \approx \frac{V}{4}\left(1-\frac{\sigma_{L}}{\sigma_{p}}\right)$ & $\frac{1}{4(m+1)} \cdot \frac{V}{V_{0}} \cdot\left(1-\frac{\sigma_{L}}{\sigma_{p}}\right)\left(\frac{\sigma_{p}-\sigma_{L}}{\sigma_{u}}\right)^{m}$ & \\
\hline \multirow[t]{4}{*}{$\sigma_{L}=0$} & Uniaxial tension & $V$ & $\frac{V}{V_{0}} \cdot\left(\frac{\sigma_{p}}{\sigma_{u}}\right)^{m}$ & $\left(\frac{\sigma_{p}}{\sigma_{0}}\right)^{m}$ \\
\hline & Pure bending & $\frac{V}{2}$ & $\frac{1}{2(m+1)} \cdot \frac{V}{V_{0}} \cdot\left(\frac{\sigma_{p}}{\sigma_{u}}\right)^{m}$ & \\
\hline & 3-point bending & $\frac{V}{2}$ & $\frac{1}{2(m+1)^{2}} \cdot \frac{V}{V_{0}} \cdot\left(\frac{\sigma_{p}}{\sigma_{u}}\right)^{m}$ & \\
\hline & 4-point bending* & $\frac{V}{2}$ & $\frac{(m+2)}{4(m+1)^{2}} \cdot \frac{V}{V_{0}} \cdot\left(\frac{\sigma_{p}}{\sigma_{u}}\right)^{m}$ & \\
\hline
\end{tabular}

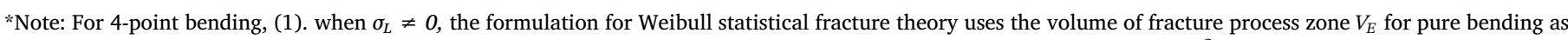

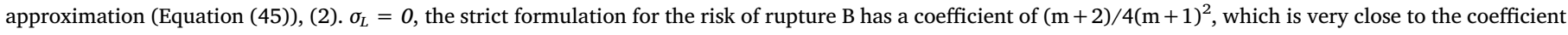
of $1 / 4(\mathrm{~m}+1)$ for 4 -point bending and $\sigma_{L} \neq 0$ when $\mathrm{m} \geq 5$.

Equation (44). In case the quantity $\left(1-\frac{\sigma_{L}}{\sigma_{p}}\right) \approx 1$ is taken, Equation (45) further reduces to the following expression similar to Equation (43):

$$
\begin{aligned}
P \approx & 1-\exp \left[-\frac{1}{4(m+1)} \cdot \frac{V}{V_{0}} \cdot\left(\frac{\sigma_{p}-\sigma_{L}}{\sigma_{u}}\right)^{m}\right]=1 \\
& -\exp \left[-\left(\frac{\sigma_{p}-\sigma_{L}}{\sigma_{0}}\right)^{m}\right] \ldots\left(\sigma_{0}=\sigma_{u} \cdot \sqrt[m]{4(m+1)\left(V_{0} / V\right)}\right)
\end{aligned}
$$

Fig. 3 compares Equations (44)-(46) against Equation (40) in combination with Equation (20) for different combinations of $\mathrm{m}=5$, $10, \mathrm{~V} / \mathrm{V}_{0}=1,1000,10000$, and $\sigma_{\mathrm{L}} / \sigma_{\mathrm{u}}=0.25,0.5$ at $\sigma_{\mathrm{u}}=600 \mathrm{MPa}$. It shows that both Equations (44) and (45) approximate to Equation (40) in combination of Equation (20) closely, but Equation (46) is much different from Equation (40). Again, Equation (46) shows the inaccuracy of using the ordinary three-parameter Weibull distribution function Equation (10) to fit uniaxial flexural strength.

Table 1 compares the ordinary Weibull distribution and the Weibull statistical fracture theory for statistics of strength in uniaxial tension and uniaxial flexure for the case of $\sigma_{\mathrm{L}} \neq 0$ and $\sigma_{\mathrm{L}}=0$, respectively. Due to the item $\left(1-\sigma_{L} / \sigma_{p}\right)$ included in the Weibull statistical fracture theory for uniaxial flexure, the result of strength fitting with the ordinary three-parameter Weibull distribution is not a close approximation to the statistics of flexural strength.

The simplified solutions to the risk of rupture (B) for fracture induced by surficial flaws for $\mathrm{m}$ as a positive integer are given in Appendix B.

For other more complex loading modes, such as biaxial flexure tests, one can expect more complicated formulation for Weibull statistical fracture theory in the case of $\sigma_{\mathrm{L}} \neq 0$, which can be much more different from the ordinary three-parameter Weibull distribution function.

\section{Assessment of strength distribution using Weibull statistical fracture theory}

The three-parameter ordinary Weibull distribution function Equation (10) only empirically fits the flexural strength data. Therefore, it is attractive to directly resort to the Weibull statistical fracture theory to infer the flaw distribution parameters $\left(m, \sigma_{L}, \sigma_{u} \cdot \sqrt[m]{V_{0}}\right)$ from sampled flexural strength data. In addition, the Weibull statistical fracture theory can be also used to synchronize specimen size effect on flexural strength for proportional size scaling, as demonstrated in Ref. [18,19].
For these two purposes, Equations (13) for uniaxial tension, (41), (42), and (45) for flexure are rewritten as the following "master curve" expressions $\left(\sigma_{N}=\sigma_{p}\right)$,

Uniaxial tension: $\frac{1}{V} \operatorname{Ln}\left(\frac{1}{1-P}\right)=\frac{1}{V_{0}}\left(\frac{\sigma_{N}-\sigma_{L}}{\sigma_{u}}\right)^{m}$

Pure bending: $\frac{1}{\frac{\left(V /(2)\left(1-\sigma_{L} / \sigma_{N}\right)\right)}{V_{E}}} \operatorname{Ln}\left(\frac{1}{1-P}\right)=\frac{1}{(m+1) V_{0}}\left(\frac{\sigma_{N}-\sigma_{L}}{\sigma_{u}}\right)^{m}$

3 - point bending: $\frac{1}{\frac{\left(V / 2\left(1-\sigma_{L} / \sigma_{N}\right)^{2}\right)}{V_{E}}} \operatorname{Ln}\left(\frac{1}{1-P}\right)=\frac{1}{(m+1)^{2} V_{0}}\left(\frac{\sigma_{N}-\sigma_{L}}{\sigma_{u}}\right)^{m}$

4 - point bending: $\frac{1}{\frac{\left(V / 4\left(1-\sigma_{L} / \sigma_{N}\right)\right)}{V_{E}}} \operatorname{Ln}\left(\frac{1}{1-P}\right)=\frac{1}{(m+1) V_{0}}\left(\frac{\sigma_{N}-\sigma_{L}}{\sigma_{u}}\right)^{m}$

where $\mathrm{V}_{\mathrm{E}}$ is the effective volume of fracture process zone in which $\sigma(x, y) \geq \sigma_{L}$ is satisfied. The dependence of master curve formulation on specimen geometry and loading mode revealed by Equations (47)-(50) leads to the three observations:

(1). It is feasible to synchronize strength data measured from different sized specimens of geometrical similarity in a same loading mode due to a same master curve formulation. This is known as the proportional size scaling [23].

(2). Due to the different master curve formulations for different loading modes, there is no straightforward approach to synchronize strength data measured from specimens of same or different geometries tested in different loading modes. This is known as the nonproportional size scaling [23].

(3). Unless the master curve formulation is known for a specific specimen with a given loading mode, which provides the explicit expression of $\mathrm{V}_{\mathrm{E}}$ as the effective volume of fracture process zone and the relationship between the empirical scale parameter $\sigma_{0}$ and the material property $\sigma_{\mathrm{u}}$, the empirical expression for strength fitting using the ordinary Weibull distribution function Equation (10) does not have prediction power for either proportional or nonproportional size scaling due to Equation (9) as elucidated in 

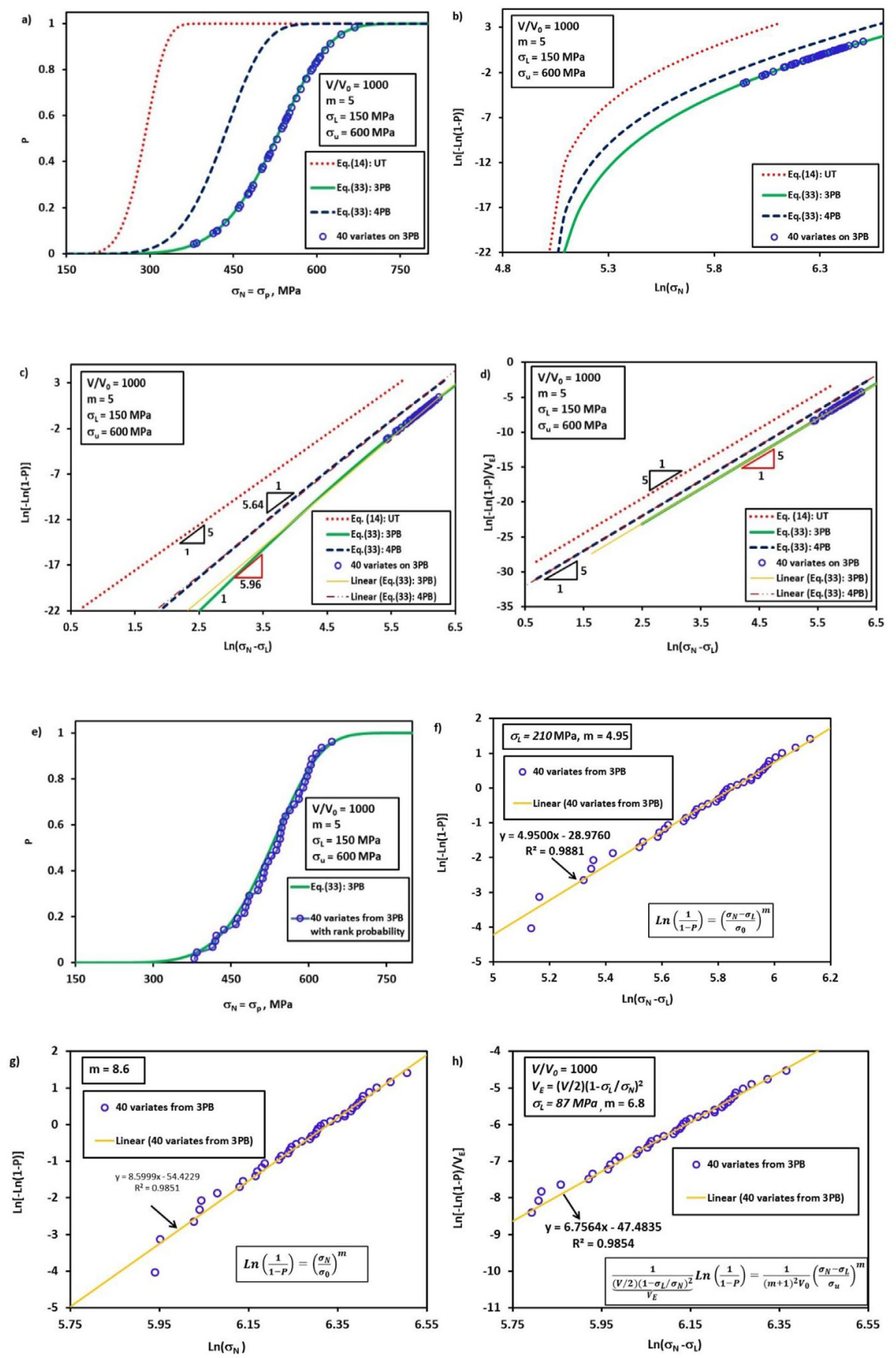

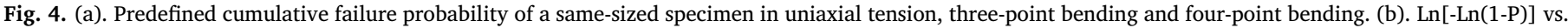

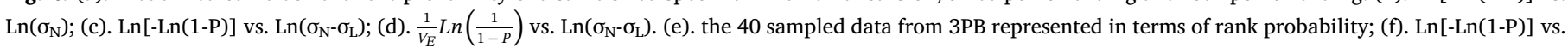

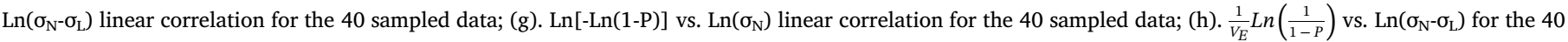
sampled data.

Equations (47)-(50).

\subsection{A numerical experiment on statistical evaluation of strength}

\subsubsection{Random variation of strength}

Fig. 4a) shows the cumulative failure probability of a same-sized specimen in uniaxial tension, three-point bending and four-point bending, respectively. The explicit formulation, Equation (33), is used to calculate the cumulative probability for the two uniaxial flexure loading modes. The model parameters are set as $\mathrm{m}=5, \sigma_{0}=600 \mathrm{MPa}$, $\sigma_{\mathrm{L}}=150 \mathrm{MPa}, \mathrm{V} / \mathrm{V}_{0}=1000$. In addition, a set of 40 randomly sampled strength data from three-point bending is also presented. There are three different ways to represent the datasets in Fig. 4a:

(1). The double-logarithmic correlation between $\operatorname{Ln}[-\operatorname{Ln}(1-\mathrm{P})]$ and $\operatorname{Ln}$ $\left(\sigma_{N}\right)$ (Fig. 4b). It shows that using an ordinary two-parameter Weibull distribution function to represent the data in Fig. 4a will inevitably show a kinky curve. Therefore, such a nonlinear 
correlation between $\operatorname{Ln}[-\operatorname{Ln}(1-\mathrm{P})]$ and $\operatorname{Ln}\left(\sigma_{\mathrm{N}}\right)$ observed on experimental data does not necessarily translate into the existence of bimodal or multimodal statistical flaw distributions in the specimens as suggested in Ref. [14], concluding the failure of Weibull statistical fracture theory.

(2). The double-logarithmic correlation between $\operatorname{Ln}[-\operatorname{Ln}(1-\mathrm{P})]$ and $\operatorname{Ln}$ $\left(\sigma_{N^{-}} \sigma_{L}\right)$ (Fig. 4 c). It shows that using an ordinary three-parameter Weibull distribution function to represent the data in Fig. 4a results in almost straight lines but with different slope values, namely $\mathrm{m}=5$ for uniaxial tension, $\mathrm{m}=5.96$ for 3 -point bending, and $\mathrm{m}=5.64$ for 4-point bending. The non-linear correlation for 3point bending does not justify the failure of Weibull statistics. Note that if only the 40 variates sampled on the 3-point bending strength distribution are fitted for the correlation, the value of $\mathrm{m}$ will be very close to 5 . This shows the effect of sample size on data fitting.

(3). The master curve-based correlation between $\frac{1}{V_{E}} \operatorname{Ln}\left(\frac{1}{1-P}\right)$ and Ln $\left(\sigma_{N^{-}} \sigma_{L}\right)$ according to Equations (47)-(50) (Fig. 4d). This ensures a constant slope value equal to its true value in each case $(m=5)$. Note that the effective volume $\mathrm{V}_{\mathrm{E}}$ varies with loading mode.

In this numerical experiment the true value of probability is known for each sampled data point. In a real experiment, each strength measurement is assigned to a rank probability. This will have more impacts on interpreting $\operatorname{Ln}[-\operatorname{Ln}(1-\mathrm{P})]$ vs. $\operatorname{Ln}\left(\sigma_{\mathrm{N}}-\sigma_{\mathrm{L}}\right)$ and $\operatorname{Ln}[-\operatorname{Ln}(1-\mathrm{P})]$ vs. $\operatorname{Ln}\left(\sigma_{\mathrm{N}}\right)$ correlations and further on estimating Weibull parameters. To elaborate the complexity with the adoption of rank probability, Fig. 4 (e) shows the same 40 variates from three-point bending specimens as shown in Fig. 4 (a)-(d) in terms of their rank probability values in comparison with the theoretical distribution curve in green. Note that by coincidence, the 40 randomly picked strength data points are on the "linear" segment in Fig. 4 (b), (c), and (d), instead of covering the "kinky" lower-shelf segment. The rank probability $P(i)$ for the i-th value of the 40 variates arranged in an ascending order is calculated by $P(i)=((i-0.3)) /((N+0.4))$, with $N=40, i=, 1,2, \ldots N$. Fig. 4 (f) shows the result of data fitting using the ordinary three-parameter Weibull distribution function. $\mathrm{m}=4.95$ and $\sigma_{\mathrm{L}}=210 \mathrm{MPa}$ are obtained for the maximum coefficient of determination $\left(R^{2}=0.9881\right)$ using the least square estimation. Fig. 4 (g) shows the result of data fitting using the ordinary two-parameter Weibull distribution function $\left(\sigma_{\mathrm{L}}=0\right) . \mathrm{m}=8.6$ is obtained for the maximum coefficient of determination $\left(\mathrm{R}^{2}=0.9851\right)$. Fig. $4(\mathrm{~h})$ shows the result of data fitting using the master curve Equation (49). $\mathrm{m}=6.76$ and $\sigma_{\mathrm{L}}=87 \mathrm{MPa}$ are obtained for the maximum coefficient of determination $\left(R^{2}=0.9854\right)$. All the three methods of data fitting yield results more or less deviated from the true values $\left(\mathrm{m}=5, \sigma_{\mathrm{L}}=150 \mathrm{MPa}\right)$, which reveals the impact of sample size $(\mathrm{N})$ on parameter estimation. Mehrvar et al. [24] studied the effect of sample size on calibrating the two-parameter ordinary Weibull distribution for fitting strength of glass polyalkenoate cements.

\subsubsection{Size effect on strength}

Fig. 5a) shows the cumulative failure probability of three differentsized, proportionally scaled specimens in three-point bending. The explicit formulation, Equation (37), is used to calculate the cumulative probability. The related model parameters are $\mathrm{m}=10, \sigma_{0}=600 \mathrm{MPa}$, $\sigma_{\mathrm{L}}=150 \mathrm{MPa}$. The volumes of the three specimens are $\mathrm{V} / \mathrm{V}_{0}=100$, 1000,10000 , respectively. In addition, a set of 15 strength data is randomly sampled from each specimen. As an alternative representation of Fig. $5 \mathrm{a}$ and $\mathrm{b}$ ) shows the master curve-based correlation between $\frac{1}{V_{E}} \operatorname{Ln}\left(\frac{1}{1-P}\right)$ and $\operatorname{Ln}\left(\sigma_{\mathrm{N}^{-}} \sigma_{\mathrm{L}}\right)$ according to Equation (65), where the true probability is adopted for each data point. However, when each sampled data point is assigned to a rank probability, the result of parameter estimation will be affected by sample size. Fig. 5c) shows the doublelogarithmic correlation between $\operatorname{Ln}[-\operatorname{Ln}(1-\mathrm{P})]$ and $\operatorname{Ln}\left(\sigma_{N}\right)$ for the samples of each specimen group based on the ordinary two-parameter Weibull distribution function. The scatter of estimated Weibull parameters is understandable in view of the sample size $(\mathrm{N}=15)$. Fig. 5d) shows the master curve-based correlation between $\frac{1}{V_{E}} \operatorname{Ln}\left(\frac{1}{1-P}\right)$ and $\operatorname{Ln}\left(\sigma_{\mathrm{N}}-\sigma_{\mathrm{L}}\right)$ according to Equation (49). The rank probability is assigned to each sampled strength datum in Fig. $5 \mathrm{c}$ ) and d). While the expected master curve correlation is verified in Fig. 5d), the linear square estimate gives $\sigma_{\mathrm{L}}=0$ instead of the true value $\sigma_{\mathrm{L}}=150 \mathrm{MPa}$, also due to the limited sample size. This reveals the difficulty in determining the threshold stress $\sigma_{\mathrm{L}}$ with limited number of data points.

\subsection{Real experimental data}

\subsubsection{Random variation of strength in a very large experimental data set}

Gorjan and Ambrožič [5] reported a statistical evaluation of 5100 experimental values of flexural strength of alumina ceramics in a series of four point bending tests. Refer to Fig. 1, the specimen sizes are: $\mathrm{L}=40 \mathrm{~mm}, \mathrm{~b}=4 \mathrm{~mm}, \mathrm{~h}=3 \mathrm{~mm}$. Therefore, $\mathrm{V}=\mathrm{bhL}=480 \mathrm{~mm}^{3}$. The master curve Equation (66) and the ordinary 2-parameter Weibull disribution function are used for linear square estimate, respectively, as shown in Fig. 6 (a) and (b) by assuming $\mathrm{V}_{0}=1 \mathrm{~mm}^{3}$. Table 2 summarizes the estimated results, including that by maximum likelihood estimate in Ref. [5]. Also the estimated values of $\sigma_{0}$ are transformed into $\sigma_{\mathrm{u}}$ according to the relationship in Table 1 for $\sigma_{L}=0$. For the ordinary 2-parameter Weibull distribution function approach, the least square estimate and the maximum likelihood estimate reached very close result.

The two sets of estimated values of Weibull parameters $\left(\sigma_{\mathrm{L}}=0\right.$, $\mathrm{m}=9.328, \sigma_{\mathrm{u}}=305.25 \mathrm{MPa}$; and $\sigma_{\mathrm{L}}=55 \mathrm{MPa}, \mathrm{m}=7.172$, $\sigma_{\mathrm{u}}=164.73 \mathrm{MPa}$ ) are used to predict the failure probability of other two different sized flexure specimens $\left(\mathrm{V} / \mathrm{V}_{0}=10 ; 10000\right)$. Specifically, according to Table 1 , the following equation is used for failure prediction using the estimated values from the ordinary 2-parameter Weibull distribution function:

$P=1-\exp \left[-\frac{(m+2)}{4(m+1)^{2}} \cdot \frac{V}{V_{0}} \cdot\left(\frac{\sigma_{N}}{\sigma_{u}}\right)^{m}\right]$

The results are shown in Fig. 7. Clearly, although the ordinary twoparameter Weibull distribution estimation shows slightly higher probability (more conservative) for fitting real experimental data than the master curve approach, it predicts a lower failure probablity (less conservative) than the latter when $\mathrm{P} \geq 10^{-4}$ for specimens of $\mathrm{V} /$ $\mathrm{V}_{0}=10$ and when $\mathrm{P} \geq 10^{-2}$ for specimens of $\mathrm{V} / \mathrm{V}_{0}=10000$. This result is against the conventional opinion that an ordinary two-parameter Weibull distribution always yields more conservative estimation (higher value) of failure probability.

The result in Fig. 7 highlights the importance to determine the threshold value $\left(\sigma_{\mathrm{L}}\right)$ in future studies.

\subsubsection{The indication of the existence of non-zero threshold stress}

Refer to Fig. 4b, for the strength data in uniaxial tension or flexure that strictly obey the three-parameter Weibull statistical fracture theory, when they are represented by the ordinary two-parameter Weibull distribution function, the double-logarithmic correlation between $\operatorname{Ln}[-\operatorname{Ln}(1-\mathrm{P})]$ and $\operatorname{Ln}\left(\sigma_{N}\right)$ inevitably becomes a kinky curve instead of a straight line. This nonlinear correlation between $\operatorname{Ln}[-\operatorname{Ln}(1-\mathrm{P})]$ and $\operatorname{Ln}\left(\sigma_{N}\right)$ is a strong indication for the existence of a non-zero threshold strength $\sigma_{L}$, rather than a deterministic signal for the failure of Weibull statistical fracture theory owing to the existence of bimodal or multimodal statistical flaw distributions in the specimens as suggested in Ref. [14]. The non-linear correlation between $\operatorname{Ln}[-\operatorname{Ln}(1-\mathrm{P})]$ and $\operatorname{Ln}\left(\sigma_{\mathrm{N}}\right)$ can be often observed in many experimental measurements of strength of quasi-brittle materials. Here below are a few examples. Fig. 8 exemplifies four published data sets of ceramic strength in threepoint bending of prismatic beams [25-28], which are presented in terms of two-parameter ordinary Weibull distribution. Fracture initiation in the feldspathic body porcelain after grinding with 100-grit SiC 

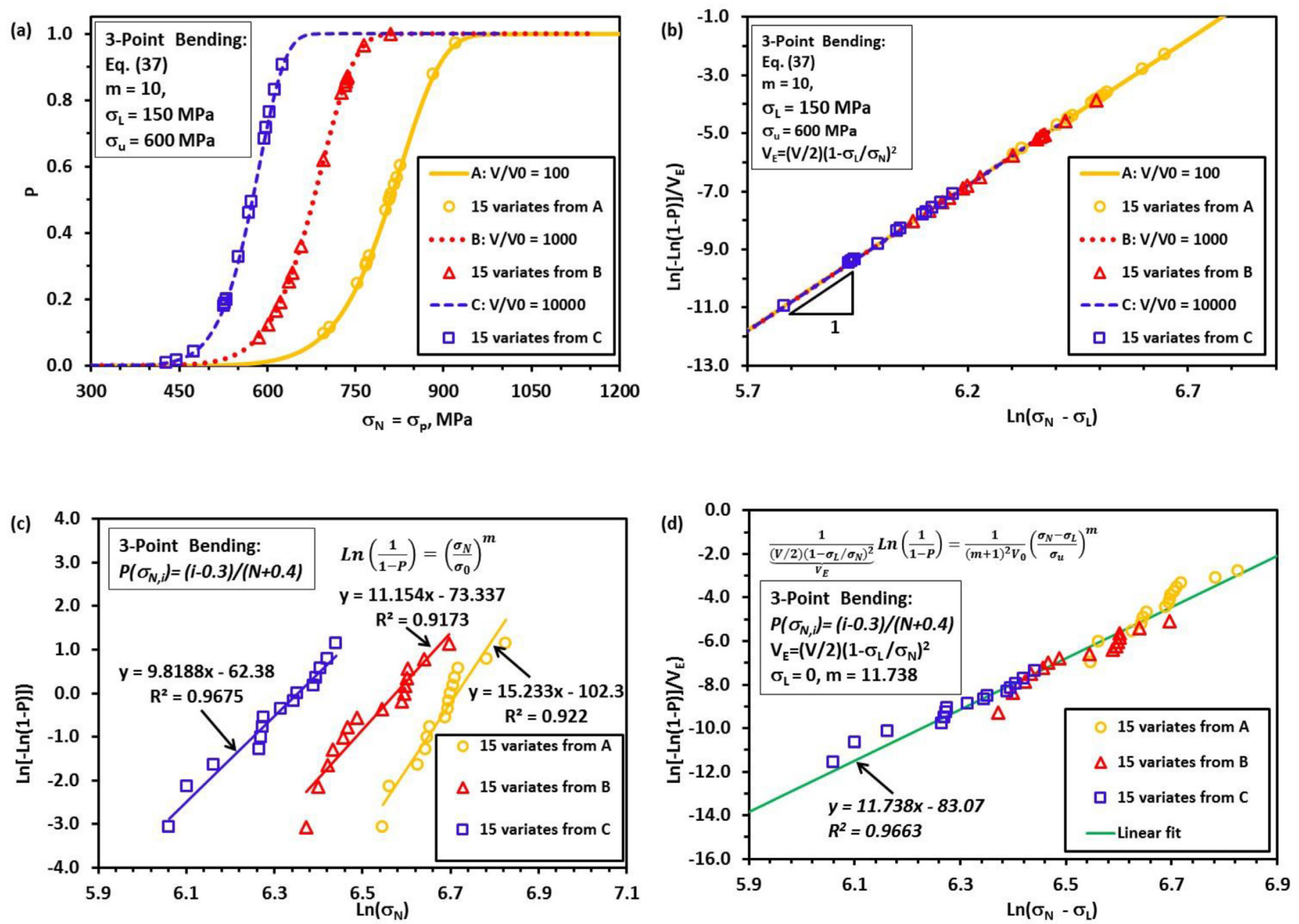

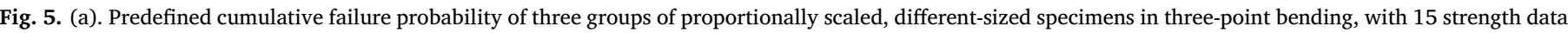

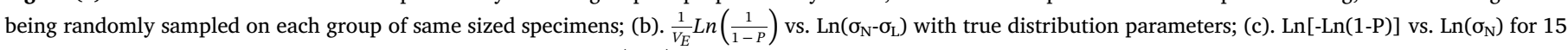
sampled data of each group of same sized specimens; (d). $\frac{1}{V_{E}} \operatorname{Ln}\left(\frac{1}{1-P}\right)$ vs. $\operatorname{Ln}\left(\sigma_{\mathrm{N}^{-}} \sigma_{\mathrm{L}}\right)$ for all 45 sampled data of three different sized specimens.
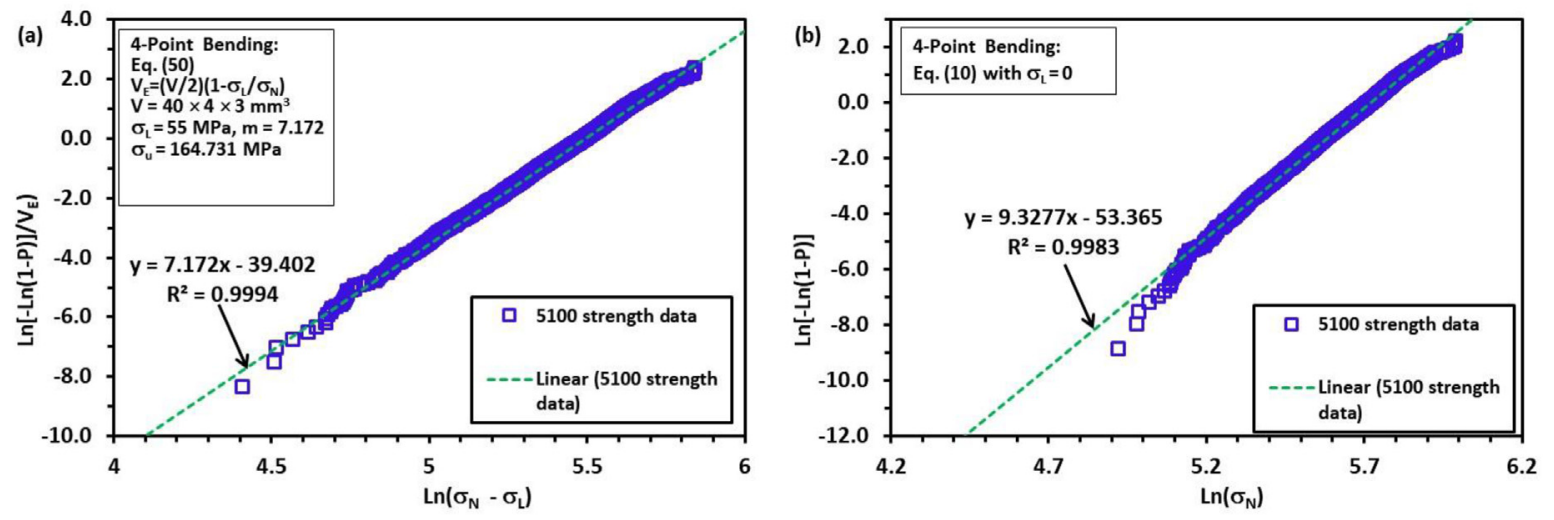

Fig. 6. Weibull parameter estimation based on the master curve Equation (50) (a) and the ordinary 2-parameter Weibull distribution function (b).

Table 2

Summary of Weibull parameter estimation using different approaches.

\begin{tabular}{|c|c|c|c|c|c|c|}
\hline Method of estimation & & $\sigma_{\mathrm{L}}, \mathrm{MPa}$ & $\mathrm{m}$ & $\sigma_{0}, \mathrm{MPa}$ & $\sigma_{\mathrm{u}}, \mathrm{MPa}$ & $\mathrm{R}^{2}$ \\
\hline \multirow[t]{2}{*}{ 2-parameter ordinary Weibull distribution function Equation (10) [5] } & Maximum likelihood & 0 & 9.048 & 305.54 & $229.86^{\mathrm{a}}$ & 0.9988 \\
\hline & Least square & & 9.328 & 305.25 & $232.37^{\mathrm{a}}$ & 0.9983 \\
\hline Master curve Equation (50) & Least square & 55 & 7.172 & / & 164.73 & 0.9994 \\
\hline
\end{tabular}

${ }^{\text {a }} \sigma_{\mathrm{u}}$ is transformed from the estimated values of $\sigma_{0}$ according to the relationship in Table 1 for $\sigma_{L}=0$. 

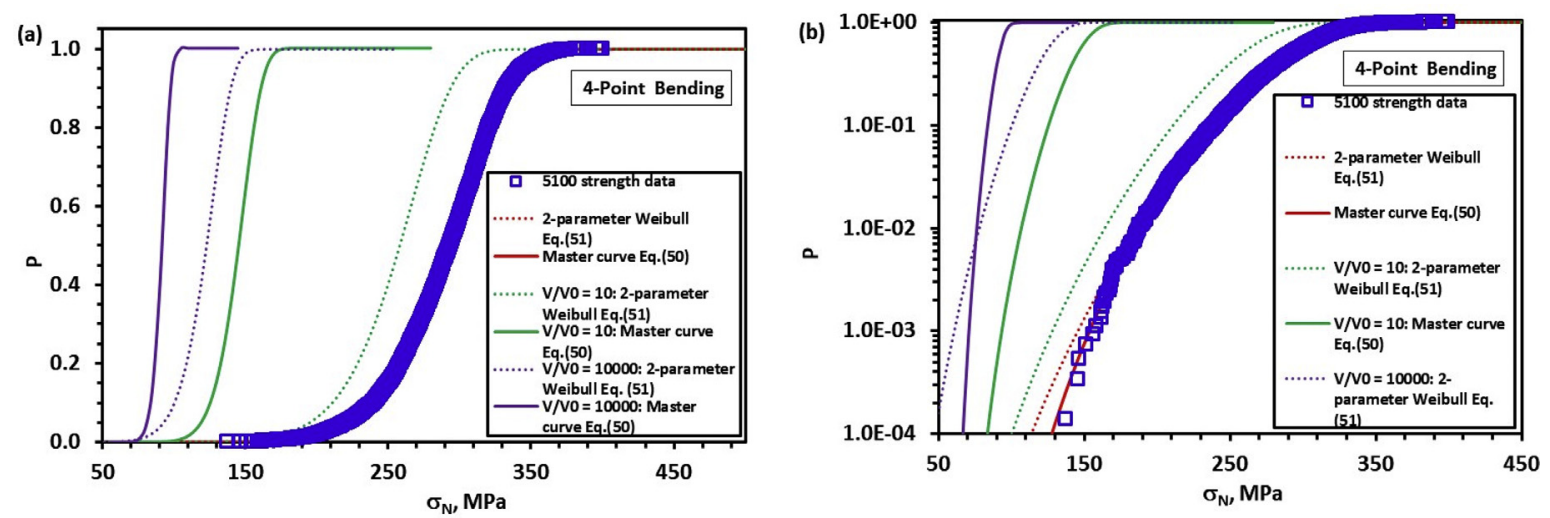

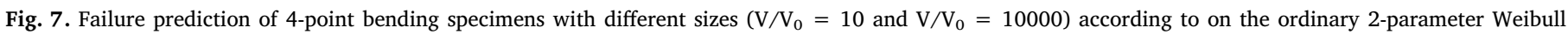
distribution function Equation (51) and the master curve Equation (50): (a). probability in linear scale and (b). probability in logarithmic scale.

abrasive was attributed to surface roughness [25]. The WZ-Zirconia [26] is a partially sintered Y-TZP ceramics $\left(\mathrm{ZrO}_{2}+\mathrm{Y}_{2} \mathrm{O}_{3}+\mathrm{HfO}_{2}\right)$, in which surface flaws were responsible for fracture initiation. Fracture initiation in both AlN ceramics [27] at low temperature and flash sintered 3\% Yttria Stabilized Zirconia [28] were due to volumetric flaws. A kinky curve is shown for all four examples, indicating a threshold behavior of fracture. The same data sets in Fig. 8 are now presented in Fig. 9 in terms of the three-parameter ordinary Weibull distribution, which all result in a linear correlation with high coefficients of determination $\left(\mathrm{R}^{2}\right)$. As highlighted earlier, when the ordinary Weibull distribution function is used to fit strength data, the value of coefficient of determination $\left(\mathrm{R}^{2}\right)$ justifies whether a two-parameter or a threeparameter function format should be used. In either case, the Weibull parameters $\left(\mathrm{m}, \sigma_{0}, \sigma_{\mathrm{L}}\right)$ are simply the fitting parameters instead of material properties. However, for strength prediction or size scaling of strength, the Weibull statistical fracture theory is needed to analyze the strength data, for which the fitted Weibull parameters $\left(\mathrm{m}, \sigma_{\mathrm{u}}, \sigma_{\mathrm{L}}\right)$ are material properties. The same data sets in Fig. 8 are now presented in Fig. 10 in terms of the Weibull statistical fracture theory.

\subsubsection{Size effect on strength}

Fischer et al. [3] studied size effect on the flexural strength of a
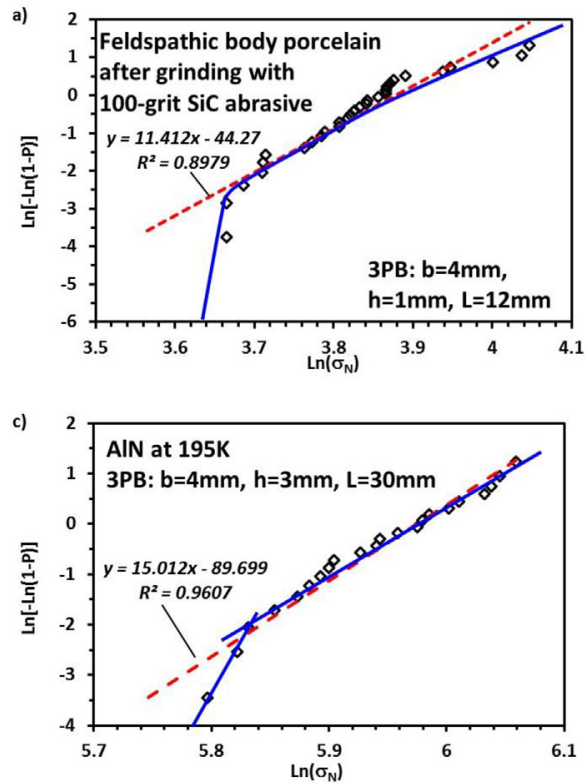

dental leucite reinforced ceramic material based on the two-parameter Weibull statistical fracture theory, which includes four sets of prismatic beams of different size in 3-point bending tests and one set in a 4-point bending test. Refer to Fig. 1, the specimen sizes are listed in Table 3.

Fig. 11 (a) shows the experimental results. Fischer et al. [3] considered the combined contribution of both volumetric and surficial microdefects to fracture. Since their SEM analysis showed the pore that induced the failure of a specimen was located inside the material but very close to the surface, Lei and Su [14] assumed that the fracture is controlled by surficial flaws and applied the weakest link formulation to study the proportional size scaling of the strength in three-point bending. Here, the following master curve equation based on Equation (B15) for fracture induced by surface flaws in 3-point bending is used for estimation of Weibull parameters:

3 - point bending: $\frac{1}{\frac{(b L)\left(1-\sigma_{L} / \sigma_{N}\right)}{A_{E}}} \operatorname{Ln}\left(\frac{1}{1-P}\right)=\frac{1}{2(m+1) A_{0}}\left(\frac{\sigma_{N}-\sigma_{L}}{\sigma_{u}}\right)^{m}$

Fig. 11b) shows the fitting result for 3-point bending strength data. According to Equation (52) the fitting line $y=5.0867 x-25.719$ translates into the final results: $\sigma_{\mathrm{L}}=34.5 \mathrm{MPa}, \mathrm{m}=5.1, \sigma_{\mathrm{u}}=96.04 \mathrm{MPa}$ due to $\operatorname{Ln}[2(\mathrm{~m}+1)]+\operatorname{mLn}\left(\sigma_{u}\right)=25.719$. Substitution of the estimated
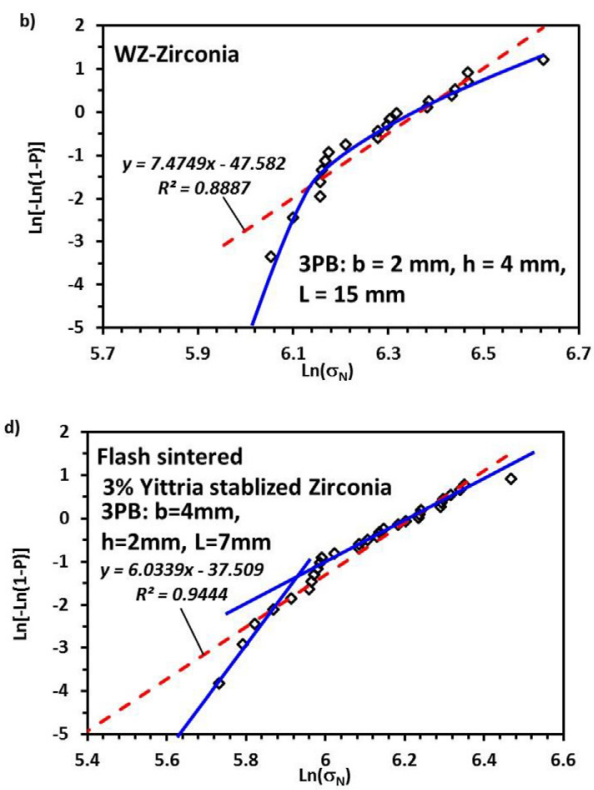

Fig. 8. Two-parameter ordinary Weibull distribution fitting of experimental strength data of different ceramic materials in three-point bending of prismatic beams: (a). Feldspathic body porcelain [25], (b). WZ-Zirconia [26] (c). AlN at 195K [27], (d). flash sintered 3\% Yttria Stabilized Zirconia [28]. 

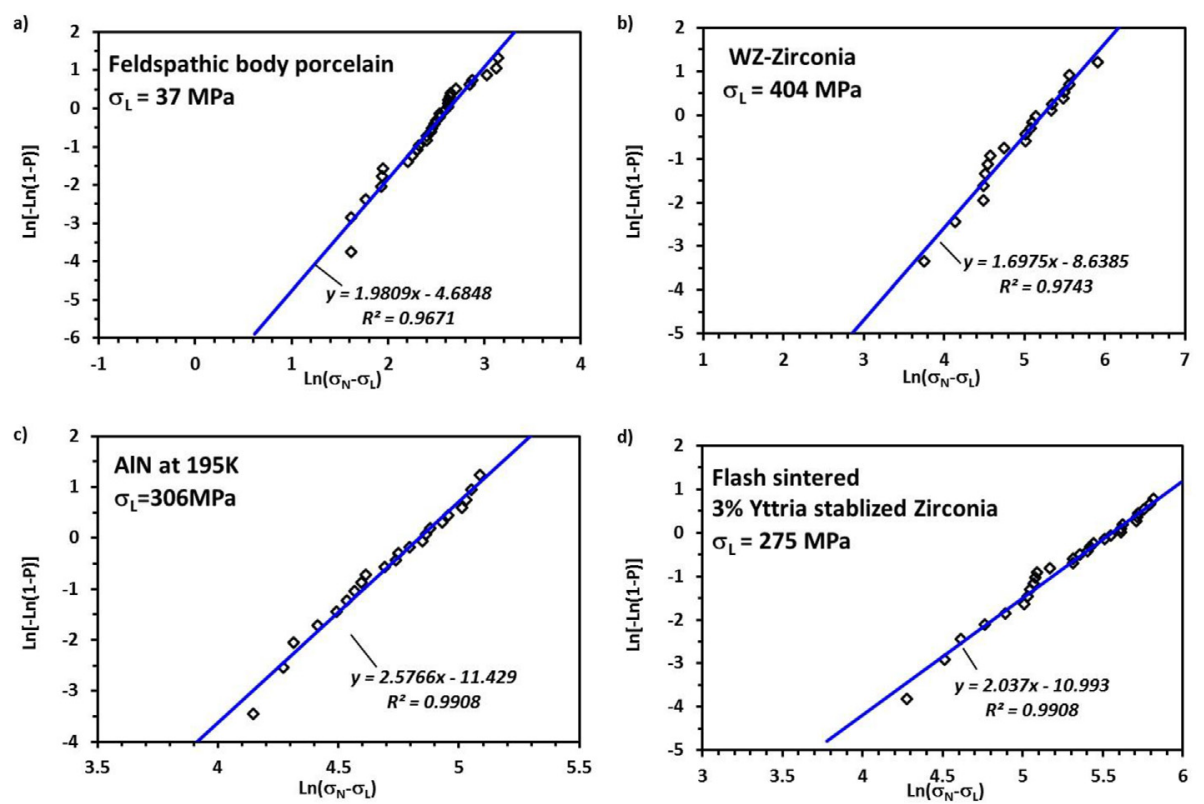

Fig. 9. Three-parameter ordinary Weibull distribution fitting of experimental strength data of different ceramic materials in three-point bending of prismatic beams: (a). Feldspathic body porcelain [25], (b). WZ-Zirconia [26] (c). AlN at 195K [27], (d). flash sintered 3\% Yttria Stabilized Zirconia [28].
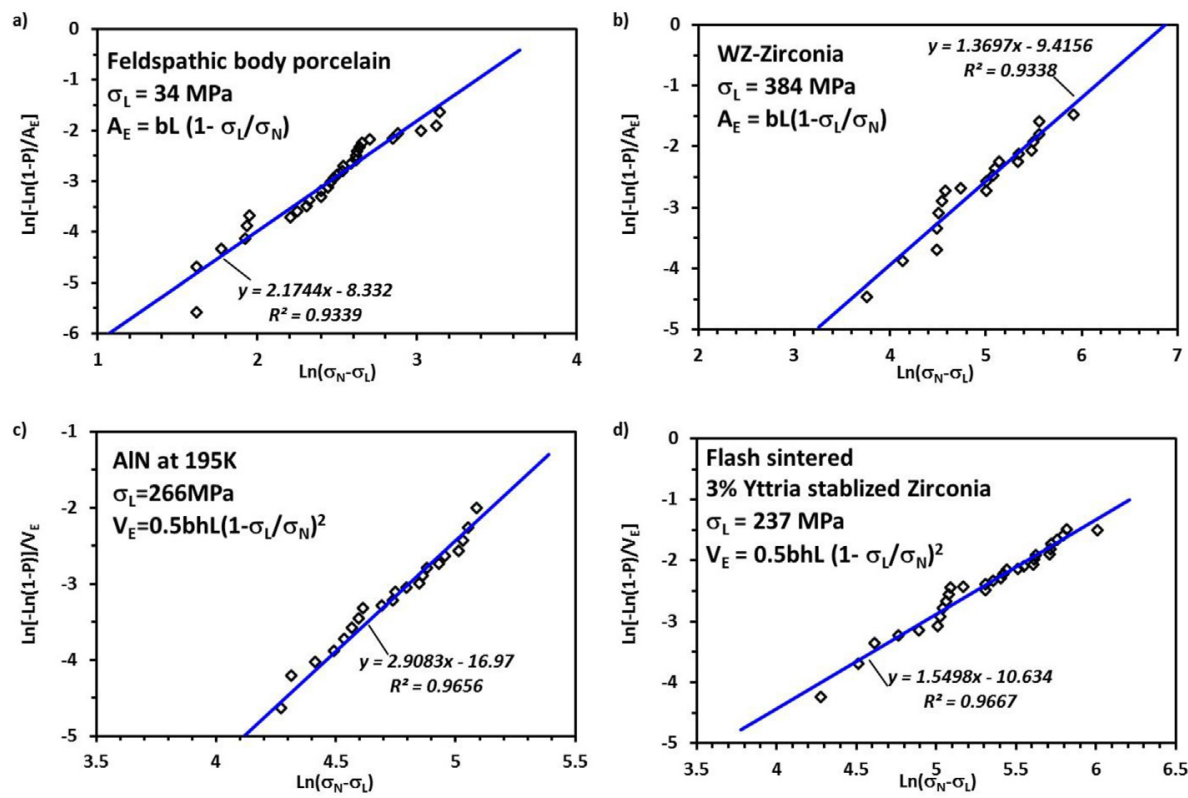

Fig. 10. Three-parameter Weibull statistical fracture theory-based analysis of experimental strength data of different ceramic materials in three-point bending of prismatic beams: (a). Feldspathic body porcelain [25], (b). WZ-Zirconia [26] (c). AlN at 195K [27], (d). flash sintered 3\% Yttria Stabilized Zirconia [28].

Table 3

Dimensions of the specimens and loading span in three- and four-point bending tests [5].

\begin{tabular}{|c|c|c|c|c|c|c|c|c|c|}
\hline Series & Number of samples & Width b, mm & Thickness $\mathrm{h}, \mathrm{mm}$ & Loading span L, mm & \multicolumn{2}{|c|}{ Reference Fischer et al. [3] } & \multicolumn{3}{|c|}{ This study $(\mathrm{A} 0=1 \mathrm{~mm} 2)$} \\
\hline 3РB-A & 15 & 2.5 & 0.9 & 12.0 & 6.4 & 117 & 5.1 & 96.0 & 34.5 \\
\hline 3РB-C & 21 & 5.9 & 5.9 & 10.0 & 6.0 & 102 & & & \\
\hline 3PB-D & 21 & 5.9 & 5.9 & 25.0 & 7.9 & 93 & & & \\
\hline 4PB-E & 18 & 6.3 & 6.0 & 24.0 & 9.0 & 77 & & & \\
\hline
\end{tabular}

*Note: For 4PB-E specimen, inner loading span is $12 \mathrm{~mm}$ (half of outer loading span $\mathrm{L}$ ). 

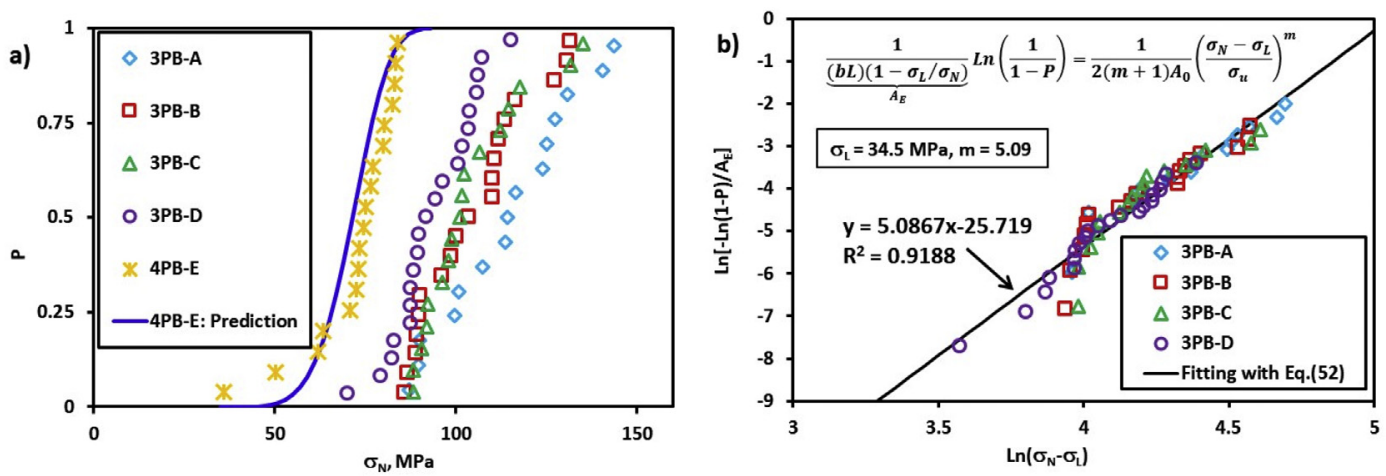

Fig. 11. The flexural strength of a dental leucite reinforced ceramic material [3](a) and Weibull parameter estimation using Equation (55) for 3-point bending specimens (b).

parameters in the following equation for fracture controlled by surface flaws in 4-point bending according to Equation (B16) in Appendix B gives the predicted failure probability at any prescribed strength, as shown by the solid line in Fig. 11a), which is close to experimental measurement:

4 - point bending: $\mathrm{P}=1$

$$
\begin{aligned}
& -\exp \left\{-\frac{L b}{2 A_{0}}\left(\frac{\sigma_{N}-\sigma_{L}}{\sigma_{u}}\right)^{m}\right. \\
& \left.\left[1+\frac{(1+h / b)}{(m+1)}\left(1-\frac{\sigma_{L}}{\sigma_{N}}\right)\right]\right\}
\end{aligned}
$$

\section{Discussions}

In literature, the term "Weibull statistics" for strength distribution may refer to either the ordinary Weibull distribution function or the Weibull statistical fracture theory. The distinction between these two concepts is emphasized in this work. Based on the preceding investigations, their relationship can be summarized in Fig. 12. The nonequivalence between the three-parameter ordinary Weibull distribution function (Equation (10)) and the three-parameter Weibull statistical fracture theory (Equation (1)) is highlighted.

The explicit expression of ordinary Weibull distribution function is unique and independent of specimen geometry and loading mode. However, the explicit expression for the Weibull statistical fracture theory depends heavily on specimen geometry and loading mode. In this work, we only reformulated the explicit expressions for the prismatic specimens in uniaxial flexure, which are quite different from the ordinary three-parameter Weibull distribution function. For other types of frequently used specimens and loading mode for strength measurement, such as the uniaxial flexure of cylindrical rods, and the biaxial flexure of circular discs, the corresponding explicit expressions are

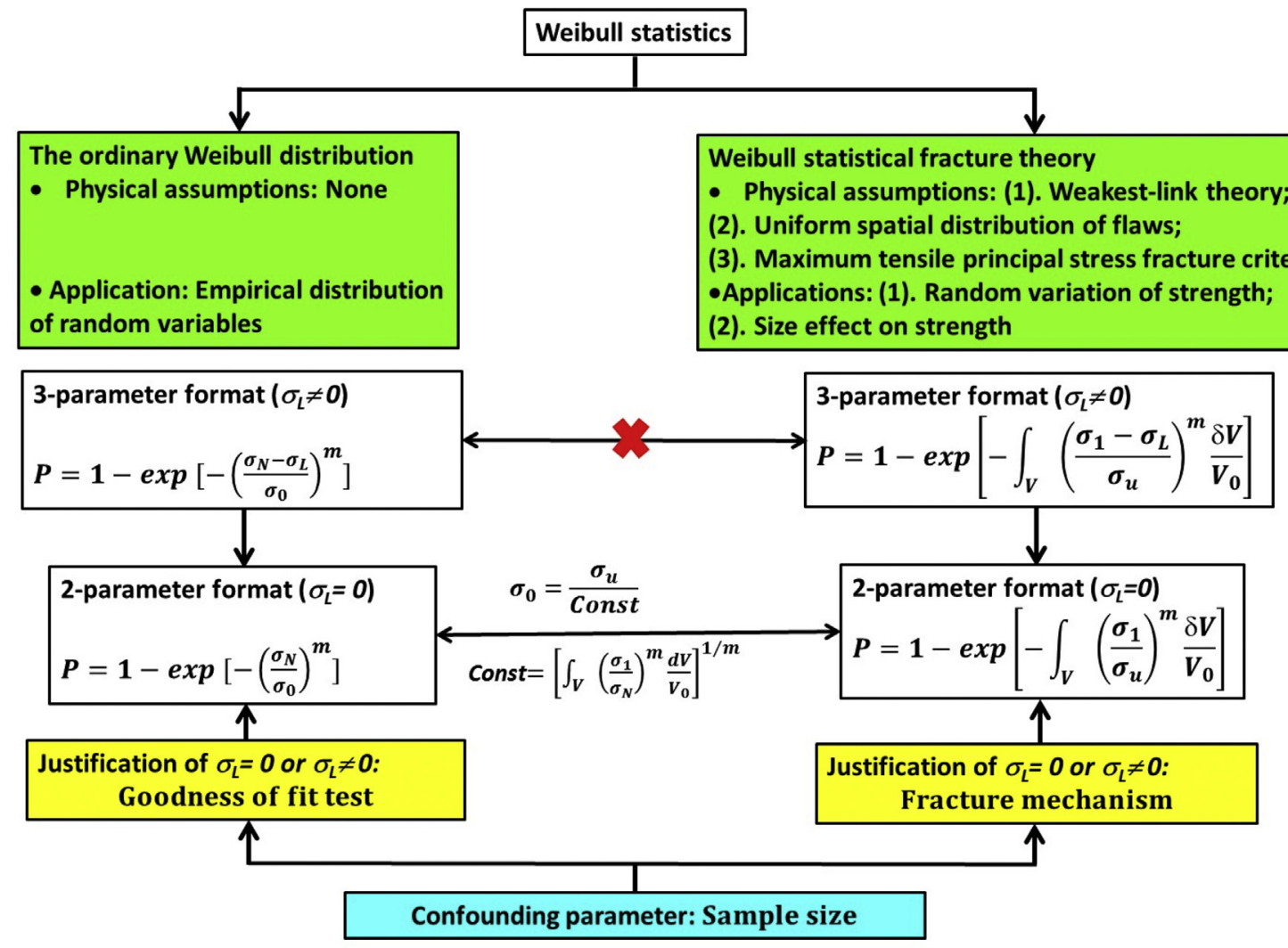

Fig. 12. The relationship between ordinary Weibull distribution function [15] and Weibull statistical fracture theory [17]. 
more complex and much more different from the ordinary three parameter Weibull distribution function. Numerical methods are needed. In such cases, using the ordinary three-parameter Weibull distribution function in Equation (10) to fit the strength data and interpret size effect on strength can be very misleading for prediction purpose.

This work has the following significance: Since uniaxial tensile experiment is often inconvenient for ceramics, and it is difficult to obtain the explicit expressions of the cumulative failure probability for specimens in more complex biaxial flexure setup, the relatively simple uniaxial flexure experiments are the practical choice for calibrating Weibull statistical fracture theory. The reformulated expressions of the cumulative failure probability for uniaxial flexural specimens ensure that the calibrated Weibull parameters are truly materials property and therefore can be used for size scaling of strength for different specimens with different loading modes and for predicting fracture behavior of real ceramic components of complex geometry and loading conditions in aid of numerical calculation.

It is worth mentioning that Equation (4) is adopted as the probability density function (PDF) for flaw distribution in a material in terms of strength (S) to ensure the mathematical self-consistency of Weibull statistical fracture theory. As emphasized in preceding studies $[1,2,7,18,19]$, the statistical distribution of flaws in a real material needs to be described with respect to four characteristics namely, their size, shape, spatial orientation, and spatial location. According to the Griffith theory $S \cdot Y(a) \sqrt{a}=C$, where $a$ is flaw size, $Y(a)$ is a factor reflecting flaw shape and loading mode, $S$ is the fracture strength, $C$ is a material constant, the distributions of flaw size and shape can be collectively represented by strength distribution $g(S)$ for simplicity. This avoids the difficulty to precisely describe the real distributions of both the shapes and the sizes of flaws. The Weibull statistical fracture theory is based on a few critical assumptions as below $[1,7,18]$ :

(1). Weakest link postulate.

(2). Uniform spatial distribution of flaws in a material;

(3). Equation (4) for flaw distribution in a material in terms of strength (S)

(4). Maximum principal tensile stress criterion for fracture occurrence.

Each of these assumptions must be carefully dissected to gain a pertinent understanding. The assumption (1) implies that Weibull statistics of strength is subject to the weakest-link assumption. In other words, Weibull statistics is not equivalent to, but is only a subordinate member of the weakest-link statistics. In the case that different assumptions in terms of spatial and strength distributions of flaws and microscopic fracture criterion other than the assumptions (2), (3) and (4) are adopted, according to the weakest-link formulation for fracture Equation (3), different expressions of cumulative failure probability will be obtained. For example, as studied in Ref. [1], the weakest-link postulate is built on the mutual independence of microcracks and is not necessarily bounded to the uniform spatial distribution of microcracks as assumption (2). By taking a power-law spatial distribution of mutually non-interactive microcracks, the following generalized weakestlink statistical formulation is obtained [1]:

$P=1-\exp \left\{\beta\left(\frac{V}{V_{0}}\right)^{\beta-1} \int_{V} \ln \left[1-p\left(\sigma, V_{0}\right)\right] \cdot \frac{\delta V}{V_{0}}\right\}$

where $\beta$ is a material constant with $\beta>0$ that describes the number $(N)$ of microcracks in a volume $V$ by $N==\left(V / V_{0}\right)^{\beta}$. With the same assumptions (3) and (4), the following extended Weibull statistical fracture theory is obtained [1]:

$P=1-\exp \left[-\beta\left(\frac{V}{V_{0}}\right)^{\beta-1} \int_{V}\left(\frac{\sigma_{1}-\sigma_{L}}{\sigma_{u}}\right)^{m} \frac{d V}{V_{0}}\right]$

Equation (55) is validated in Ref. [1,2,7,9] for ceramics, concrete and some other quasi-brittle materials.

In [19], it is shown that the strength distribution $g(S)$ in Equation (4) is equivalent to the flaw size distribution below if all flaws are penny shaped microcracks:

$f(a)=\frac{m}{2 a} \cdot \sqrt{\frac{a_{0}}{a}} \cdot\left(\sqrt{\frac{a_{0}}{a}}-\sqrt{\frac{a_{0}}{a_{u}}}\right)^{m-1} \cdot \exp \left[-\left(\sqrt{\frac{a_{0}}{a}}-\sqrt{\frac{a_{0}}{a_{u}}}\right)^{m}\right]$

where $a_{u}$ is the maximum flaw size corresponding to the threshold strength $\sigma_{\mathrm{L}}, \mathrm{a}_{0}$ is the characteristic flaw size corresponding to the scale parameter $\sigma_{\mathrm{u}}$, both according to the Griffith law.

However, the flaw distribution in a real material may be different from Equation (4) as adopted as assumption (3). The work [7] reports that when the flaw size $(a)$ distribution $f(a)$ is described by the power law, $f(a)=\kappa a^{-b}$, where $b$ and $\kappa$ are material constants, under the same assumptions (1), (2), (4), the cumulative failure probability has much complicated expressions as below:

$P=1-\exp \left\{\int_{V} \ln \left[1-\frac{\ln \left(\sigma_{1} / \sigma_{L}\right)}{\ln \left(\sigma_{u} / \sigma_{L}\right)}\right] \frac{d V}{V_{0}}\right\} \cdot(b=1)$

$P=1-\exp \left\{\int_{V} \ln \left[\left(\frac{\sigma_{L}}{\sigma_{1}}\right)^{m}\right] \frac{d V}{V_{0}}\right\}(0<b<1, m=2 b-2)$

$P=1-\exp \left[\int_{V} \ln \left(\frac{\sigma_{u}^{m}-\sigma_{1}^{m}}{\sigma_{u}^{m}-\sigma_{L}^{m}}\right) \frac{d V}{V_{0}}\right] \ldots(b>1, m=2 b-2)$

In terms of assumption (4), the maximum tensile stress criterion essentially ignores the effect of flaw orientation. Weibull [13] did extend his theory to the multiaxial stress states as below by adopting a normal tensile stress criterion $\sigma_{n} \geq S$, where $\sigma_{n}$ is the normal tensile stress component acting on a microcrack surface. $\sigma_{n}$ is a function of three principal stresses and microcrack orientation.

$P=1-\exp \left\{-\int_{V}\left[\int_{\Omega=4 \pi}\left(\frac{\sigma_{n}-\sigma_{L}}{\sigma_{u}}\right)^{m} \frac{d \Omega}{4 \pi}\right] \frac{d V}{V_{0}}\right\} \ldots\left(\sigma_{n} \geq 0\right)$

Note that Equation (58) assumes the spatial orientation of microcracks in terms of the solid angle $(\Omega)$ of the normal vector takes a uniform distribution over the whole solid angle $\Omega=4 \pi$. The assumption of uniform spatial orientation makes the mathematical description much simpler while it may not be always approximately true for real materials. Both the maximum tensile stress criterion and the normal tensile stress criterion may not be pertinent to the variety of stress conditions in real structural components including pure compression and multiaxial stress states. For example, they are unable to interpret the failure of material under uniaxial compression. The work [2] found that the shear stress criterion can reasonably explain the size effect of uniaxial compressive strength of concrete. The work [29] summarized different equivalent stress $\left(\sigma_{\mathrm{e}}\right)$ criteria for fracture initiation $\sigma_{e} \geq S$ to account for multiaxial stress effects.

To sum up, the Weibull statistical fracture theory in Equation (1) is subject to several critical assumptions which may oversimplify the flaw distribution and multiaxial stress effect in a real material. Nevertheless, its relatively simple expression leads to its common adoption in academic studies. Meanwhile, there have been constant efforts to develop more advanced statistical fracture theory to address growing challenges in reliability design of ceramic components in engineering.

\section{Conclusions}

(1). The essential difference between the ordinary Weibull distribution function and Weibull statistical fracture theory is interpreted. The ordinary Weibull distribution function is an empirical distribution function for many different variables in addition to brittle fracture strength. It can be used for strength data fitting for a given 
specimen but not for size scaling of strength, since the fitted Weibull parameters are not material properties.

(2). Weibull statistical fracture theory is a subordinate member of the weakest-link statistics under the assumption of uniform spatial distribution of flaws, in which the Weibull parameters are material properties. It has the prediction power and can be used for size scaling of strength and transference of strength data between different specimen geometries and loading modes.

(3). The three-parameter Weibull statistical fracture theory for uniaxial flexure of prismatic beams is reformulated and validated by numerical experiments and real strength data of different ceramics.

\section{Author contributions}

WSL: Conceptualization, Methodology, Formal analysis, Validation, Writing-Original draft, Review \& Editing, Data curation; PZ: Methodology, Validation, Investigation, Writing-Review \& Editing; ZY:
Methodology, Investigation, Resources, Writing-Review \& Editing. GQ: Methodology, Validation, Visualization, Writing - Review \& Editing.

\section{Declaration of competing interest}

The authors declare no conflict of interest.

\section{Acknowledgement}

The author is indebted to Dr. Gorjan of Jožef Stefan Institute, Ljubljana, Slovenia, for generously sharing his large data set of ceramic flexural strength which is cited and analyzed in this study. GQ is grateful to the funding of National Natural Science Foundation of China (No.11872364) and Chinese Academy of Science (CAS) Pioneer Hundred Talents Program.

\section{Appendix A}

The expressions of $\mathbf{U}(\mathbf{m})=\sum_{i=0}^{m} T^{i} \sum_{j=0}^{i}\left(\begin{array}{l}\boldsymbol{i} \\ j\end{array}\right) \frac{(-1)^{i-j}}{\boldsymbol{m}+\boldsymbol{j}-\boldsymbol{i}+1}$ at $\mathrm{m}=1,2, \ldots, 10$

$\mathrm{U}(\mathrm{m}=1)=\frac{1}{2}-\frac{T}{2}$

$\mathrm{U}(\mathrm{m}=2)=\frac{1}{3}-\frac{T}{6}+\frac{T^{2}}{3}$

$\mathrm{U}(\mathrm{m}=3)=\frac{1}{4}-\frac{T}{12}+\frac{T^{2}}{12}-\frac{T^{3}}{4}$

$\mathrm{U}(\mathrm{m}=4)=\frac{1}{5}-\frac{T}{20}+\frac{T^{2}}{30}-\frac{T^{3}}{20}+\frac{T^{4}}{5}$

$\mathrm{U}(\mathrm{m}=5)=\frac{1}{6}-\frac{T}{30}+\frac{T^{2}}{60}-\frac{T^{3}}{60}+\frac{T^{4}}{30}-\frac{T^{5}}{6}$

$\mathrm{U}(\mathrm{m}=6)=\frac{1}{7} \pm \frac{T}{42}+\frac{T^{2}}{105}-\frac{T^{3}}{140}+\frac{T^{4}}{105}-\frac{T^{5}}{42}+\frac{T^{6}}{7}$

$\mathrm{U}(\mathrm{m}=7)=\frac{1}{8}-\frac{T}{56}+\frac{T^{2}}{168}-\frac{T^{3}}{280}+\frac{T^{4}}{280}-\frac{T^{5}}{168}+\frac{T^{6}}{56}-\frac{T^{7}}{8}$

$\mathrm{U}(\mathrm{m}=8)=\frac{1}{9}-\frac{T}{72}+\frac{T^{2}}{252}-\frac{T^{3}}{504}+\frac{T^{4}}{630}-\frac{T^{5}}{504}+\frac{T^{6}}{252}-\frac{T^{7}}{72}+\frac{T^{8}}{9}$

$\mathrm{U}(\mathrm{m}=9)=\frac{1}{10}-\frac{\mathrm{T}}{90}+\frac{\mathrm{T}^{2}}{360}-\frac{\mathrm{T}^{3}}{840}+\frac{\mathrm{T}^{4}}{1260}-\frac{\mathrm{T}^{5}}{1260}+\frac{\mathrm{T}^{6}}{840}-\frac{\mathrm{T}^{7}}{360}+\frac{\mathrm{T}^{8}}{90}-\frac{\mathrm{T}^{9}}{10}$

$\mathrm{U}(\mathrm{m}=10)=\frac{1}{11}-\frac{T}{110}+\frac{T^{2}}{495}-\frac{T^{3}}{1320}+\frac{T^{4}}{2310}-\frac{T^{5}}{2772}+\frac{T^{6}}{2310}-\frac{T^{7}}{1320}+\frac{T^{8}}{495}-\frac{T^{9}}{110}+\frac{T^{10}}{11}$

\section{Appendix B. Risk of rupture for fracture induced by surficial flaws}

Refer to Fig. 1, for fracture controlled surficial flaws, Weil and Daniel [22] obtained

$B=\frac{L}{(m+1) A_{0}}\left(1-\frac{\sigma_{L}}{\sigma_{p}}\right)\left(\frac{\sigma_{p}-\sigma_{L}}{\sigma_{u}}\right)^{m}\left[\left(1-\frac{2}{k}\right) h+\frac{2 h}{k} \sum_{r=0}^{[m]} \frac{1}{m+1+r}\left(1-\frac{\sigma_{p}}{\sigma_{L}}\right)^{-r}+\frac{2 b}{k}\right]+\left(1-\frac{2}{k}\right)\left(\frac{\sigma_{p}-\sigma_{L}}{\sigma_{u}}\right)^{m} \frac{L b}{A_{0}}+\frac{2 h L I(\alpha)}{k(m+1) V_{0}} \frac{\left(-\sigma_{L}\right)^{[m]+1}}{\sigma_{p} \sigma_{u}^{m}}$

If $m$ is an integer, Eq. (B1) reduces to

$B=\frac{L}{(m+1) A_{0}}\left(1-\frac{\sigma_{L}}{\sigma_{p}}\right)\left(\frac{\sigma_{p}-\sigma_{L}}{\sigma_{u}}\right)^{m}\left[\left(1-\frac{2}{k}\right) h+\frac{2 h}{k} \sum_{r=0}^{[m]} \frac{1}{m+1+r}\left(1-\frac{\sigma_{p}}{\sigma_{L}}\right)^{-r}+\frac{2 b}{k}\right]+\left(1-\frac{2}{k}\right)\left(\frac{\sigma_{p}-\sigma_{L}}{\sigma_{u}}\right)^{m} \frac{L b}{A_{0}}+\frac{2 h L}{k(m+1) A_{0}} \frac{\left(-\sigma_{L}\right)^{[m]+1}}{\sigma_{p} \sigma_{u}^{m}} \ln \left(\frac{\sigma_{p}}{\sigma_{L}}\right)$

We now simplify Equation (B2) (for $\mathrm{m}$ as a positive integer). There is

$B=\int_{A}\left(\frac{\sigma-\sigma_{L}}{\sigma_{u}}\right)^{m} \frac{d A}{A_{0}}=B_{1 s}+B_{2 s}+B_{3 s}+B_{4 s}$

With 
$B_{1 s}=\frac{2 b}{A_{0}} \int_{T L / k}^{L / k}\left(\frac{\frac{k \sigma_{p}}{L} x-\sigma_{L}}{\sigma_{u}}\right)^{m} d x$

$B_{2 s}=\frac{(1-2 /(k) L) b}{A_{0}}\left(\frac{\sigma_{p}-\sigma_{L}}{\sigma_{u}}\right)^{m}$

$B_{3 s}=\frac{4}{A_{0}} \int_{T h / 2}^{h / 2} d y \int_{x_{\min }}^{x_{\max }}\left(\frac{\frac{2 k \sigma_{p}}{h L} y x-\sigma_{L}}{\sigma_{u}}\right)^{m} d x$

$B_{4 s}=\frac{2(1-2 /(k) L)}{A_{0}} \int_{T h / 2}^{h / 2}\left(\frac{\frac{2 \sigma_{p}}{h} y-\sigma_{L}}{\sigma_{u}}\right)^{m} d y$

It can be obtained that

$B_{1 s}=\frac{2 b L}{k(m+1) A_{0}}\left(1-\frac{\sigma_{L}}{\sigma_{p}}\right)\left(\frac{\sigma_{p}-\sigma_{L}}{\sigma_{u}}\right)^{m}$

$B_{2 s}=\frac{(k-2) L b}{k A_{0}}\left(\frac{\sigma_{p}-\sigma_{L}}{\sigma_{u}}\right)^{m}$

$B_{3 s}=\frac{2 h L}{k(m+1) A_{0}}\left(1-\frac{\sigma_{L}}{\sigma_{p}}\right)^{2}\left(\frac{\sigma_{p}-\sigma_{L}}{\sigma_{u}}\right)^{m} \sum_{i=0}^{m} T^{i} \sum_{k=0}^{i}\left(\begin{array}{l}i \\ k\end{array}\right) \frac{(-1)^{i-k}}{m+k-i+1}$

$B_{4 s}=\frac{(k-2) L h}{k(m+1) A_{0}}\left(1-\frac{\sigma_{L}}{\sigma_{p}}\right)\left(\frac{\sigma_{p}-\sigma_{L}}{\sigma_{u}}\right)^{m}$

Therefore,

$B=\left(\frac{\sigma_{p}-\sigma_{L}}{\sigma_{u}}\right)^{m}\left[\frac{(k-2) L b}{k A_{0}}+\frac{2 b L+(k-2) h L}{k(m+1) A_{0}}\left(1-\frac{\sigma_{L}}{\sigma_{p}}\right)+\frac{2 h L}{k(m+1) A_{0}}\left(1-\frac{\sigma_{L}}{\sigma_{p}}\right)^{2} \sum_{i=0}^{m} T^{i} \sum_{k=0}^{i}\left(\begin{array}{l}i \\ k\end{array}\right) \frac{(-1)^{i-k}}{m+k-i+1}\right]$

or

$B=\left(\frac{\sigma_{p}-\sigma_{L}}{\sigma_{u}}\right)^{m}\left\{\frac{(k-2) L b}{k A_{0}}+\frac{2 b L+(k-2) h L}{k(m+1) A_{0}}\left(1-\frac{\sigma_{L}}{\sigma_{p}}\right)+\frac{2 h L}{k(m+1)^{2} A_{0}}\left(1-\frac{\sigma_{L}}{\sigma_{p}}\right)^{2}\left[1+O\left(\frac{1}{m(m+1)^{2}} \cdot \frac{\sigma_{L}}{\sigma_{p}}\right)\right]\right\}$

For $k=\infty$ (pure bending), Eq. (B13) reduces to

$B=\frac{L b}{A_{0}}\left(\frac{\sigma_{p}-\sigma_{L}}{\sigma_{u}}\right)^{m}+\frac{h L}{(m+1) A_{0}}\left(1-\frac{\sigma_{L}}{\sigma_{p}}\right)\left(\frac{\sigma_{p}-\sigma_{L}}{\sigma_{u}}\right)^{m}$

For $k=2$ (three-point bending), there is

$B \approx\left(\frac{\sigma_{p}-\sigma_{L}}{\sigma_{u}}\right)^{m}\left[\frac{b L}{(m+1) A_{0}}\left(1-\frac{\sigma_{L}}{\sigma_{p}}\right)+\frac{h L}{(m+1)^{2} A_{0}}\left(1-\frac{\sigma_{L}}{\sigma_{p}}\right)^{2}\right] \approx \frac{b L}{(m+1) A_{0}}\left(1-\frac{\sigma_{L}}{\sigma_{p}}\right)\left(\frac{\sigma_{p}-\sigma_{L}}{\sigma_{u}}\right)^{m}$

For $k=4$ (four-point bending), there is

$B \approx\left(\frac{\sigma_{p}-\sigma_{L}}{\sigma_{u}}\right)^{m}\left[\frac{L b}{2 A_{0}}+\frac{(b+h) L}{2(m+1) A_{0}}\left(1-\frac{\sigma_{L}}{\sigma_{p}}\right)+\frac{h L}{2(m+1)^{2} A_{0}}\left(1-\frac{\sigma_{L}}{\sigma_{p}}\right)^{2}\right] \approx \frac{L b}{2 A_{0}}\left(\frac{\sigma_{p}-\sigma_{L}}{\sigma_{u}}\right)^{m}\left[1+\frac{(1+h / b)}{(m+1)}\left(1-\frac{\sigma_{L}}{\sigma_{p}}\right)\right]$

Especially, when $\sigma_{\mathrm{L}}=0$, Eq. (B13) is simplified as

$B=\left(\frac{\sigma_{p}}{\sigma_{u}}\right)^{m}\left[\frac{(k-2) L b}{k A_{0}}+\frac{2 b L+(k-2) h L}{k(m+1) A_{0}}+\frac{2 h L}{k(m+1)^{2} A_{0}}\right]$

\section{References}

[1] W.-S. Lei, A generalized weakest-link model for size effect on strength of quasibrittle materials, J. Mater. Sci. 53 (2018) 1227-1245.

[2] W.-S. Lei, G. Qian, Z. Yu, F. Berto, Statistical size scaling of compressive strength of quasi-brittle materials incorporating specimen length-to-diameter ratio effect, Theor. Appl. Fract. Mech. 104 (2019) 102345, https://doi.org/10.1016/j.tafmec. 2019.102345 .

[3] H. Fischer, W. Rentzsch, R. Marx, A modified size effect model for brittle nonmetallic materials, Eng. Fract. Mech. 69 (2002) 781-791.
[4] F. Schweitzer, S. Spintzyk, J. Geis-Gerstorfer, F. Huettig, Influence of minimal extended firing on dimensional, optical, and mechanical properties of crystalized zirconia-reinforced lithium silicate glass ceramic, J. Mech. Behav. Biomed. Mater. 104 (2020), https://doi.org/10.1016/j.jmbbm.2020.103644.

[5] L. Gorjan, M. Ambrožič, Bend strength of alumina ceramics: a comparison of Weibull statistics with other statistics based on very large experimental data set, J. Eur. Ceram. Soc. 32 (2012) 1221-1227.

[6] J. Malzbender, R.W. Steinbrech, Threshold fracture stress of thin ceramic components, J. Eur. Ceram. Soc. 28 (2008) 247-252.

[7] W.-S. Lei, Statistical size scaling of ceramic strength, J. Am. Ceram. Soc. 102 (2019) 90-97. 
[8] E. Pirhonen, L. Moimas, M. Brink, Mechanical properties of bioactive glass9-93 fibres, Acta Biomater. 2 (2006) 103-107.

[9] G. Qian, W.-S. Lei, Z. Yu, F. Berto, Statistical size scaling of breakage strength of irregularly-shaped particles, Theor. Appl. Fract. Mech. (2019) 51-58.

[10] S. Nohut, C. Lu, Fracture statistics of dental ceramics: discrimination of strength distributions, Ceram. Int. 38 (2012) 4979-4990.

[11] W. Weibull, A statistical distribution function of wide applicability, J. Appl. Mech. 18 (1951) 293-297.

[12] J.B. Quinn, G.D. Quinn, A practical and systematic review of Weibull statistics for reporting strengths of dental materials, Dent. Mater. 26 (2010) 135-147.

[13] W. Weibull, A statistical theory of the strength of materials, 151 (1939) 1-45 Ingeniorsvetenskapakademiens. Handlinger.

[14] W.-S. Lei, Y. Su, An approach to scaling size effect on strength of quasi-brittle biomedical materials, J. Mech. Behav. Biomed. Mater. 62 (2016) 428-432.

[15] B. Deng, D. Jiang, J. Gong, Is a three-parameter Weibull function really necessary for the characterization of the statistical variation of the strength of brittle ceramics? J. Eur. Ceram. Soc. 38 (2018) 2234-2242.

[16] J. Smart, B.C. Mitchell, S.L. Fok, B.J. Marsden, The effect of the threshold stress on the determination of the Weibull parameters in probabilistic failure analysis, Eng. Fract. Mech. 70 (2003) 2559-2567.

[17] F. Kern, H. Reveron, J. Chevalier, R. Gadow, Mechanical behaviour of extremely tough TZP bioceramics, Mech. Behav. Biomed. Mater. 90 (2019) 395-403.

[18] W.-S. Lei, Evaluation of the basic formulations for the cumulative probability of brittle fracture with two different spatial distributions of microcracks, Fatig. Fract. Eng. Mater. Struct. 39 (2016) 611-623.

[19] W.-S. Lei, A framework for statistical modeling of plastic yielding initiated cleavage fracture of structural steels, Philos. Mag. A 96 (2016) 3586-3631.

[20] M. Asadollahzadeh, R. Torkaman, M. Torab-Mostaedi, J. Safdari, Using maximum entropy, Gamma, Inverse Gaussian and Weibull approach for prediction of drop size distribution in a liquid-liquid extraction column, Chem. Eng. Res. Des. 117 (2017) 637-647.

[21] M.H. Soulouknga, S.Y. Doka, N. Revanna, N. Djongyang, T.C. Kofane, Analysis of wind speed data and wind energy potential in Faya-Largeau, Chad, using Weibull distribution, Renew. Energy 121 (2018) 1-8.

[22] N.A. Weil, I.M. Daniel, Analysis of fracture probabilities in nonuniformly stressed brittle materials, J. Am. Ceram. Soc. 47 (1964) 268-274.

[23] W.-S. Lei, Z. Yu, A statistical approach to scaling size effect on strength of concrete incorporating spatial distribution of flaws, Construct. Build. Mater. 122 (2016) $702-713$.

[24] C. Mehrvar, D.J. Curran, A.M.F. Alhalawani, D. Boyd, M. Towler, Comparative study of Weibull characteristic strength and mean strength of GPCs to confirm the minimum number of samples needed for confident strength reporting, J. Mech. Behav. Biomed. Mater. 43 (2015) 53-58.

[25] Y. Nakamura, S. Hojo, H. Sato, The effect of surface roughness on the Weibull distribution of porcelain strength, Dent. Mater. J. 29 (2010) 30-34.

[26] E. Siarampi, E. Kontonasaki, L. Papadopoulou, N. Kantiranis, T. Zorba, K.M. Paraskevopoulos, P. Koidis, Lexural strength and the probability of failure of cold isostatic pressed zirconia core ceramics, J. Prosthet. Dent 108 (2012) 84-95.

[27] S. Wei, Z. Xie, W. Xue, Z. Yi, J. Chen, L. Cheng, Strengthening mechanism of aluminum nitride ceramics from 293 to 77 K, Mater. Lett. 119 (2014) 32-34.

[28] J.G. Pereira da Silva, A.N. Yamcheloua, A. Debris, C. Wiecka, H. Jelittoa, H.A. All Al-Qureshi, R. Janssen, Mechanical strength and defect distributions in flash sintered 3YSZ, J. Eur. Ceram. Soc. 37 (2017) 2901-2905.

[29] W.-S. Lei, A cumulative failure probability model for cleavage fracture in ferritic steels, Mech. Mater. 93 (2016) 184-198. 